\title{
Predicting Stability of a Decentralized Power Grid Linking Electricity Price Formulation to Grid Frequency Applying an Optimized Data-Matching Learning Network to Simulated Data
}

\author{
David A. Wood ${ }^{1}$ (D) \\ Received: 9 May 2019 / Accepted: 11 December 2019 / Published online: 4 January 2020 \\ (C) Springer Nature Singapore Pte Ltd. 2020
}

\begin{abstract}
The stability of decentralized electricity grids is influenced by real-time electricity prices and the cost sensitivity and reaction times of power producers and consumers. The decentral smart grid control (DSGC) system is designed to provide demand-side control of decentralized electricity grids by linking real-time electricity prices to changes in grid frequency over the time scale of a few seconds. This stimulates electricity demand-side consumption / production on similar time scales. Grid stability of DSGC systems can be simulated by considering a wide range of assumptions for the electricity volumes consumed / produced (P) by each grid participant, their cost-sensitivity $(\mathrm{G})$ and reaction times (Tau) to changing grid conditions. Such a simulation $(10,000$ cases) published for a simple four-node star decentralized grid configuration with randomized values for P, G and Tau quantifies dynamic grid stability $\left(S t b_{i n}\right)$ in terms of grid mechanical and pricing influences. This study applies an optimized data-matching machine-learning algorithm, the, transparent open box (TOB) learning network to predict $S t b_{\text {in }}$ (ranging from -0.0808 to $+0.1094 \mathrm{~s} \mathrm{~s}^{-2}$ ) for this published simulation from its independent variables. TOB manages to predict $S t b_{\text {in }}$ to a high degree of accuracy (RMSE $\sim 0.016 \mathrm{~s}^{-2} ; \mathrm{R}^{2} \sim 0.85$ ) for this grid configuration in which independent variables $\mathrm{P}, \mathrm{G}$ and Tau are poorly correlated with $S t b_{i n}$. By involving average $\mathrm{G}$ and Tau values for the three consumers as input variables TOB prediction accuracy is further improved (RMSE $\sim 0.0075 \mathrm{~s}^{-2} ; \mathrm{R}^{2} \sim 0.90$ ). The study highlights the importance of compound feature selection when predicting grid stability of decentralized electricity grids.
\end{abstract}

Keywords Decentralized power grids · Demand response electricity pricing · Grid stability prediction - TOB learning network . Electricity price elasticity $\cdot$ Price response flexibility

\section{Introduction}

The ability to effectively influence demand response in decentralized electricity grids without requiring expensive changes to grid infrastructure is a high-priority goal for the power sector striving to accommodate more embedded and intermittent generators and prosumers with fluctuating production and consumption. For demand-side grid control methods to be successful they must demonstrate their ability to maintain grid stability in terms of rapid changes in

Electronic supplementary material The online version of this article (https://doi.org/10.1007/s40866-019-0074-0) contains supplementary material, which is available to authorized users.

David A. Wood

dw@dwasolutions.com

1 DWA Energy Limited, Lincoln, UK electricity prices and different degrees of price sensitivity and reaction times to price changes displayed by power consumers and producers. Demand-side control systems for decentralized power grids can be simulated considering various grid configurations. Such simulations can incorporate details of the grid mechanics, electricity pricing and the response characteristics of the participant to calculate grid stability under a wide range of conditions.

This study takes the novel approach of applying a datamatching machine- learning algorithm, that does not utilize correlations, to predict simulated grid stability from three key variables, viz. the volumes of electricity consumed / produced $(\mathrm{P})$ by each grid participant, and the cost-sensitivity $(\mathrm{G})$ and reaction times (Tau) of the grid participants to changing grid conditions. The ability to accurately predict grid stability from such variables and to identify to which of those variables grid stability is most sensitive is an important capability in designing effective decentralized grid control mechanisms. 
The hypothesis being tested by this study is that a transparent data-matching, machine-learning algorithm can provide highly accurate predictions of grid stability and useful insight to the relative influences of the various input variables in contributing to grid stability of decentralized electricity grids.

The recently developed machine-learning algorithm, the transparent open box (TOB) learning network [1] is a datamatching, supervised learning algorithm that does not rely upon correlations between its input variables, prioritizing transparency and accuracy as its primary objectives. The main objectives of this study are to demonstrate the TOB's effectiveness in evaluating virtual simulations of decentralized grid performance, to predict the stability of those grids with meaningful accuracy and to provide useful insights to the relative contributions of the input variables influencing grid stability.

The expansion of decentralized power networks relies partly on embedded and distributed generation [2], with both conventional and renewable contributions, and involves new complexities such as prosumers [3]. Such networks routinely have to cope with intermittency of some power supply. Designing and adapting grid topologies in many regions to incorporate these features has become the new reality for modern power network participants in smart grids. In such grids supply-demand imbalances and fluctuation are commonplace on a range of timeframes beginning with milliseconds, making it difficult to maintain dynamic stability [4] and potentially leading to power-price instability [5]. This requires a different approach to the design and control of decentralized grids to that traditionally associated with centralized systems, crucially introducing short-term flexibility [6]. Such flexibility needs to be attainable in a cost-effective and efficient manner that is, from a pricing perspective, transparent to all grid participants not just the main power producers, suppliers and system operators [7].

Demand-side management controlling /influencing consumers' power consumption decision by encouraging them to respond to market conditions (available supply) through pricing signals is widely considered as potentially providing viable routes to the required flexibility via smart meters $[8,9]$. Demand-side response programs related to micro-grids are the focus of much recent research attention [10-13]. However, pricing routes require rapid information flow and communication systems that protect systems from cyber violations and personal data from being compromised, which comes at significant cost if a centralized mindset for control prevails [14-16]. In order to simplify the information to be communicated between electricity consumers, producers and system operators, Schafer et al. [6] introduced the Decentral Smart Grid Control (DSGC) mechanism exploiting the wellestablished ability to re-schedule power dispatch adapting to frequency fluctuations $[17,18]$.

DSGC enables the momentary supply / demand of prosumers in a smart grid demand to be linked to local measurements of frequency within the grid on a timescale of very few seconds. As fluctuations in grid frequency are typically responses to under- or over-supply of electricity within a grid (frequency decreases when there is a shortfall of power in the grid), monitoring grid frequency offers a cheap way to potentially control demand responses [19]. One way to do this is with distributed intelligent load controllers [20], especially with prosumers with loads that are flexible with regard to timing [21]. Schafer et al. [6, 22] established that DSGC could enhance dynamic power stability provided that the grid frequency was measured over sufficient time intervals and that it could potentially be applied in both centralized and decentralized grids. DSGC also can be configured to limit non-gaussian power grid frequency fluctuations [23]. Arzamasov et al. [24] conducted a simulation sampling the key variables over a broad feasible solution space to further assess grid stability with DSGC applied to a simple decentralized grid configuration. Their dataset is registered in the UCI machine-learning repository [25] and is the dataset further evaluated by this study. Other reinforcement learning approaches involving simulation of multiple agents are also applied to grid system decision and control [26-28].

Machine learning algorithms are able to spot patterns and anomalies in datasets and can therefore be deployed to potentially enable grid system operators to make real-time decisions about how to best allocate the electricity available. Various machine-learning and data- mining algorithms have been applied to the decentralized management and control of microgrids and to aid reinforcement learning [29]. These include fuzzy Q-learning [30]; artificial neural networks for conducting virtual field tests [31]; decision trees [24, 32]; support vector machines [33] for predicting transient grid instability; and, deep learning [34, 35] algorithms applied to large-scale smart-grid databases. Several studies focus machine learning studies on the detection of smart grid data compromised by cyberattacks (e.g.., [36, 37]). Transparency is typically not the priority for most machine learning algorithms as they generally are based on correlations or statistical relationships among variables.

Following this introduction, the study is presented as follows: section 2 defines and quantifies the decentral smart grid control (DSGC) system; section 3 describes the TOB machine-learning algorithm and how it is applied to the DSGC stability dataset; section 4 describes the TOB grid stability prediction results and the influence of key input variables; section 5 discusses the significance of the results and future requirements for future simulations on larger, more complex decentralized grid configurations. and section 6 presents the conclusions. Appendix 1 summarizes the steps involved and application of the TOB method. Appendix 2 describes access to the DSGC dataset compiled for this study. A terminology section following the appendices lists and defines all symbols and abbreviations used in the study. 


\section{Decentralised and Idealised Power Grid Defined and Quantified}

The physical dynamics of electric power generation and its connection with consumption loads is expressed for the DSGC model as Eq. (1) $[6,22,24]$ taking into account power grid synchronization $[38,39])$ :

$\frac{d^{2} \theta_{j}}{d t^{2}}=P_{j}-\alpha_{j} \frac{d \theta_{j}}{d t}+\sum_{k} K_{j k} \sin \left(\theta_{k}-\theta_{j}\right)$

Where:

$J \quad$ is an index representing the number of grid participant (producer(s) generating power to transmit through the grid and consumers take load from the grid);

$\frac{d^{2} \theta_{j}}{d t^{2}} \quad$ is a grid stability indicator (negative indicates grid is unstable; positive indicates grid is stable);

$P_{j} \quad$ is the mechanical power produced (e.g. by generator $P_{1}$ ) or consumed (e.g. by one of several consumers $P_{2}$ to $\left.P_{4}\right)\left(s^{-2}\right)$

$\alpha_{j} \quad$ is a damping constant related to the power dynamics of the grid;

$\frac{d \theta_{j}}{d t} \quad$ change in rotor angle for participant $j$ relative to grid frequency $\omega$;

$K_{j k} \quad$ Coupling strength between grid participant $j$ and $k$, which is proportional to line capacity $\left(s^{-2}\right)$; and,

$\theta_{j}$ and $\quad$ are rotor angles for grid participants $j$ and $k$ at a

$\theta_{k} \quad$ specific point in time $t$.

A mechanism to bind the electricity price to the DSGC, proposed by Schäfer et al. [6] involves a proportionality factor $c_{1}$, allows grid participants to adjust their production or consumption in response to price changes within a short time window of a few seconds. Electricity price for grid participant $j$ is defined as Eq. (2):

$p_{j}=p_{\omega}-c_{1} \cdot \int_{t-T_{j}}^{t} \frac{d \theta_{j}}{d t}\left(t-\tau_{j}\right) d t$

Where:

$p_{j} \quad$ is the electricity price for grid participant $j$;

$p_{\omega} \quad$ is the electricity price when $\frac{d \theta_{j}}{d t}=0$, i.e., no net change in electricity is flowing through the grid;

$c_{1}$ is a proportionality factor;

$\tau_{j}$ is a grid participants reaction time (in seconds) to execute their change in production or consumption in response to an electricity price change;

$T_{j}$ is the time interval (up to about $4 \mathrm{~s}$ ) used to define electricity prices, and is the period over which the average grid frequency is measured $\left(T_{j}\right.$ is referred to as the averaging time); and.

$t$ refers to a specific point in time.
The power produced or consumed by grid participant $\mathrm{j}$ for a specific electricity price can then be approximated by the price relationships expressed by Eq. (2) in the relationship defined by Eq. (3):

$P_{j} \approx \widehat{P}_{j}-c_{j}\left(p_{j}-p_{\omega}\right)$

Where:

$\widehat{P}_{j} \quad$ is the power produced or consumed by grid participant $j$ at electricity price $p_{j}$; and,

$c_{j} \quad$ is a coefficient proportional to the price elasticity of grid participant $j$.

The dynamic measure of grid stability for the DSGC configured in the way described is then defined by substituting $P_{j}$ in Eq. (1) with $\widehat{P}_{j}$ derived from Eq. (3) and the electricity prices $\left(p_{j}\right)$ from Eq. (3) defined by Eq. (2).

$$
\begin{aligned}
\frac{d^{2} \theta_{j}}{d t^{2}}= & P_{j}-\alpha_{j} \frac{d \theta_{j}}{d t} \\
& +\sum_{k} K_{j k} \sin \left(\theta_{k}-\theta_{j}\right)-\frac{\gamma_{j}}{T_{j}}\left(\theta_{j}\left(t-\tau_{j}\right)-\theta_{j}\left(t-\tau_{j}-T_{j}\right)\right)
\end{aligned}
$$

Where, $\gamma_{j}=c_{1} \cdot c_{j}$ and represents a coefficient proportional to price elasticity, which would vary from one grid participant to another. Eq. (4) effectively couples the mechanical and pricing influences on stability of the DSGC grid which is quantified in terms of $\frac{d^{2} \theta_{j}}{d t^{2}}$ such that a negative value for that metric indicates the grid is stable, whereas a positive number indicates that it is unstable.

Arzamasov et al. [24] used Eq.(4) to conduct a simulation to evaluate DSGC stability under a range of feasible values for ranges of input assumptions applied to a simplistic four node start motif DSGC network as illustrated in Fig. 1.

They explored the dynamic stability of the grid assuming linear stability during steady-state operations of electricity flowing from the one producer to the three consuming participants. This involved finding a matrix of roots for a set of four equations (i.e., one equation in the set for each participant in the grid, each configured as Eq. (4)). There is a vast array of possible solutions for this set of equations, depending on the input assumptions, but only some solutions will yield positive real parts to their roots, and such conditions, when they occur, determine the grid to be unstable. There are a significant number of assumptions in the simple DSGC grid simulated relating to the mechanical performance of the grid configuration and these are discussed in detail by Arzamasov et al. [24].

To conduct the simulation three key variables were consider (each allowed to vary independently for each of the four grid participants. These three key variable are: $P_{j}$ (mechanical power produced/ consumed, with $j=1$ designated as the producer and $j=2$ to 4 designated as the electricity consumers); $\gamma_{j}$ 


\section{Four Node Star Grid Simulated with Decentral Smart Grid Control}

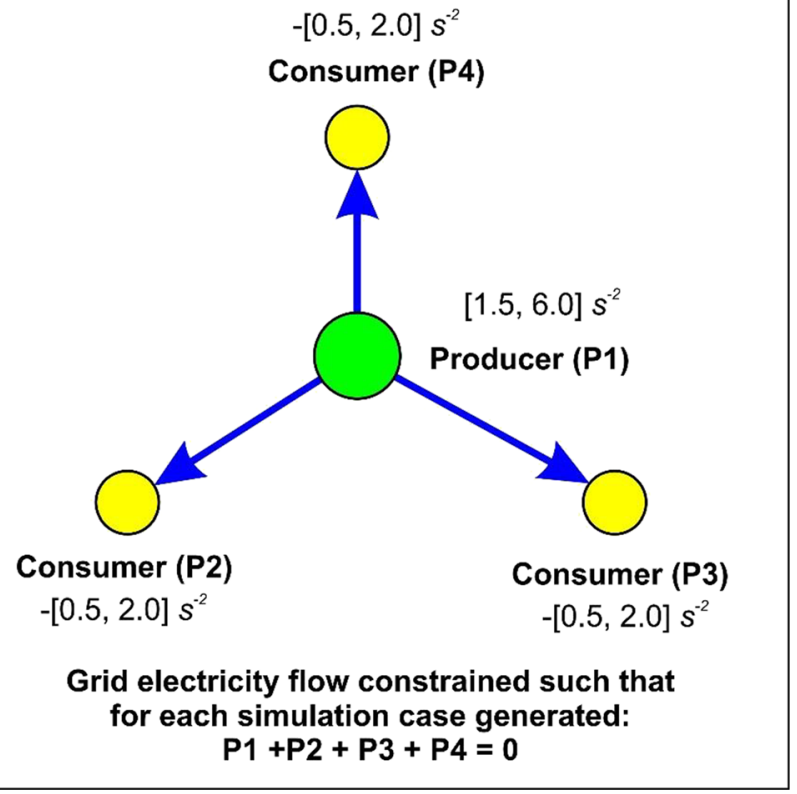

Fig. 1 Four-node star motif DSGC design and electricity flow simulated (after [24]; Schafer et al., 2016) indicating the ranges of consumption sampled as uniform distributions for grid participants P2 to P4 (consumers) for each simulation case and the $\mathrm{P} 1$ production value assigned such that $\mathrm{P} 1=-(\mathrm{P} 2+\mathrm{P} 3+\mathrm{P} 4)$

(coefficient proportional to price elasticity for each grid participant, also referred to as $\mathrm{G}$ ); and, $\tau_{j}$ (each grid participants reaction time to an electricity price change, also referred to as Tau). The feasible solution space values (boundary conditions) for these three key variables considered for Arzamasov et al.'s simulation are listed in Table 2 along with assumptions for some key constants associated with the equations presented.

The simulation model therefore involved twelve dependent variables (Tau1, Tau2, Tau3, Tau3, P1, P2, P3, P4, G1, G2, G3, G4) as defined in Table 1 together with one dependent variable, viz. $\frac{d^{2} \theta_{j}}{d t^{2}}$, the grid stability indicator calculated using Eq. (4), also referred to as $S t b_{i n}$ ). Note that two-thirds of the independent variables are electricity-price sensitive. Arzamasov et al. [24] sampled these variables, throughout their respective feasible spaces, using a random Latin hypercube simulation (LHS) design with no specific dependencies applied among the variables (dependent or independent) [40, 41], viz. participants $j=1$ to 4 were allowed to vary independently of each other throughout the constrained (Table 1) feasible spaces. Ten thousand trials were executed and $S t b_{i n}$ was calculated for each case run.

Arzamasov et al. [24] have deposited the dataset for this simulation with the UCI Machine Learning Repository [25] in November, 2018. They explored the data set with Classification and Regression Trees (CART), using that machine-learning technique in the manner developed by Breiman [42] to establish a number of useful characterization rules for the specific DSGC simulated. For example, the dataset reveals that stable grid systems lie almost exclusively at or below a Tau average value of 3.1 and a $G$ average value of less than 0.67 . They present decision tree diagrams to display a number of such boundary conditions for "stable" $\left(S t b_{i n}<=0\right)$ and "unstable" $\left(S t b_{i n}>0\right)$ grid conditions for this DSGC, correctly classifying design points with an accuracy of about $80 \%$.

That DSGC dataset is used in this study to further explore the relationships between its variables using the transparent open box (TOB) learning network. Moreover, statistical analysis reveals how the TOB method establishes its predictions of $S t b_{\text {in }}$ from its dependent variables and the prevailing relationships between them. The analysis highlights how the subtle correlations between grid participants and their respective variables might be exposed and exploited in better managing and controlling DSGC from a commercial perspective, i.e., efficient price management and price discovery for real DSGC grids in the future.

\section{Method}

\section{Applying the Optimized Nearest-Neighbour TOB Learning Network to DSGC Stability Dataset}

The TOB learning network is a recently introduced machinelearning algorithm successfully applied to small and mediumsized datasets $[1,43]$. Key benefits of the TOB algorithm are that it does not employ hidden correlations among the variables and enables forensic access to the data predictions that it makes for each data record. These attributes make the TOB algorithm able to provide useful insights to datasets as well as generate credible and transparent predictions of dependent variables that avoid overfitting. The mathematical basis and detailed implementation steps for the TOB algorithm is described in Appendix 1.

The TOB algorithm is comprised of 14 easy-to-execute and well-defined steps that are configured to provide a two-stage implementation. TOB Stage 1 involves a data matching process between a small tuning subset of data records and a large training subset. TOB Stage 2 applies an optimizer and variable weights to the matches identified in TOB Stage 1 to minimize the root mean square error (RMSE) of its predictions. The TOB Stage 1 data matching algorithm establishes the top ten $(\mathrm{Q}=10)$ matching records in the training subset to each specific record in a tuning subset. It does this by calculating the sum of the squared errors (SSE) between all the independent variables with equal weights applied. The data records in the training subset with the closest matches contribute most to the predictions TOB Stage 2 takes the top-ten matches identified by TOB Stage 1 and uses some or all of them ( $Q$ is allowed to 
Table 1 Input assumptions for the grid stability simulation conducted by Arzamasov et al. [24] for a four-node star motif DSGC design Input Variable Values \& Distributions for Four-node Star DSGC Simulation

\begin{tabular}{|c|c|c|c|c|c|c|}
\hline & Variable & Equation Symbols & $\begin{array}{l}\text { Simulation } \\
\text { Abbreviations }\end{array}$ & $\begin{array}{l}\text { Selected value or } \\
\text { distribution range } \\
\text { for simulation }\end{array}$ & Unit & Comment \\
\hline \multirow[t]{4}{*}{$\begin{array}{l}\text { Grid Infrastructure } \\
\text { Factors }\end{array}$} & $\begin{array}{l}\text { Mechanical power } \\
\text { produced }\end{array}$ & $P^{j=1}$ & P I & $(1.5 .8)$ & $\mathrm{S}^{\prime}$ & $\begin{array}{l}\text { Constrained by: } \\
\text { PI + P2 + PS.P4 a } 0\end{array}$ \\
\hline & Mechanical power consumed & $\mathrm{P}^{\mathrm{j}}$ & $\mathrm{P} 2$ to $\mathrm{P} 4$ & 40.5.2) & $S^{4}$ & \\
\hline & $\begin{array}{l}\text { Damping constant } \\
\text { (dynamic power) }\end{array}$ & $a^{j}$ & alpha 01 & 0.1 & $S^{4}$ & $\begin{array}{l}\text { In real grids could } \\
\text { vary F-0.1,-11 }\end{array}$ \\
\hline & $\begin{array}{l}\text { Coupling strength } \\
\text { between grid participants) } \\
\text { and } k\end{array}$ & $\mathrm{~K}^{\mathrm{jk}}$ & Simulation constant & 8 & S4 & $\begin{array}{l}\text { In real grids could } \\
\text { vary }(-4 .-12)\end{array}$ \\
\hline \multirow[t]{3}{*}{$\begin{array}{l}\text { Electricity Pricing } \\
\text { Factors }\end{array}$} & $\begin{array}{l}\text { Grid participant } j s \text { coefficient } \\
\text { of price elasticity }\end{array}$ & $v^{j}$ & $\mathrm{Cl}$ to $\mathrm{G} 4$ & $(0.05 .1]$ & $3^{4}$ & \\
\hline & $\begin{array}{l}\text { Grid participant } I s \text { reaction } \\
\text { time to price change }\end{array}$ & $\mathrm{t}^{\mathrm{j}}$ & Taut to Tau4 & $(0.5 .10)$ & $\mathrm{S}$ & $\begin{array}{l}\text { Upper limit could } \\
\text { be lowered for } \\
\text { some gilds }\end{array}$ \\
\hline & $\begin{array}{l}\text { Time interval used to } \\
\text { define electricity prices }\end{array}$ & $T$ & Averaging time constant & 2 & $\mathrm{~S}$ & $\begin{array}{l}\text { In real grids } T \text { code' } \\
\text { vary }(0.4)\end{array}$ \\
\hline
\end{tabular}

vary between 2 and 10) and the optimizer is allowed to apply variable weights ( 0 to 1$)$ to the squared errors associated with each independent variable.

For small data sets (up to a few thousand data records) TOB Stage 2 can be efficiently conducted using Excel's Solver optimizers. For mid-sized and large datasets, a fully coded customised optimizer is applied. For the simulated DSGC dataset both approaches are utilized. A fully-coded memetic firefly optimizer [43], with six memes or metaheuristics configured specifically for the TOB algorithm, and Excel's Solver optimizers are both applied to verify the outcomes for the tuning subsets with up to 100 data records. For larger tuning subsets just the memetic firefly optimizer is applied. An independent testing subset is used to verify that the optimum solutions derived by applying the two stages of the TOB algorithm to the tuning subset provide dependent-variable predictions that are statistically valid when applied to the dataset more generally. The two-stage process makes prediction results available for TOB Stage 1 and Stage 2 and this helps to identify and avoid overfitting datasets.

\section{Prediction Performance Measures Assessed}

Several statistical measurements of prediction accuracy are determined to monitor the prediction performance of the TOB learning network's for the grid-stability dependent-variable in the DSGC dataset. These statistical measures, and components used in their calculation, are expressed in Eq. (5) to Eq. (12), where $X_{i}$ refers to the measured value and $Y_{i}$ the TOB predicted value of a data record $i$ in the subset being considered.

\section{Mean Square Error (MSE)}

$M S E=\frac{1}{n} \sum_{i=1}^{n}\left(\left(X_{i}\right)-\left(Y_{i}\right)\right)^{2}$

\section{Root Mean Square Error (RMSE)}

$R M S E=\sqrt{\mathrm{MSE}}$

RMSE calculated with Eq. (5) and Eq. (6) is used as the objective function of the TOB algorithm.

\section{Percent Deviation between Measured and Predicted Values for Data Set Record I (PDi)}

$P D_{i}=\frac{X_{i}-Y_{i}}{X_{i}} \times 100$

\section{Average Percent Deviation (APD)}

$A P D=\frac{\sum_{i=1}^{n} P D_{\mathrm{i}}}{\mathrm{n}}$

$A P D$ combines both positive and negative percent deviations Eq. (7) and is expressed in percentage terms.

\section{Absolute Average Percent Deviation (AAPD)}

$A A P D=\frac{\sum_{i=1}^{n}\left|P D_{\mathrm{i}}\right|}{\mathrm{n}}$ 
$A A P D$ combines the absolute values of the percent deviations Eq.(7). and is also expressed in percentage terms.

\section{Standard Deviation (SD)}

$S D=\sqrt{\frac{\sum_{i=1}^{n}\left(D_{\mathrm{i}}-\text { Dimean }\right)^{2}}{\mathrm{n}-1}}$

Where:

$D i \quad$ is $\left(X_{i}-Y_{i}\right)$ for each $\left(i^{t h}\right)$ data record of a dataset; and,

Dimean is the mean of the $D i$ values of all the data records in a dataset

Dimean $=\frac{1}{n} \sum_{i=1}^{n}\left(X_{i}-Y_{i}\right)$

\section{Correlation Coefficient (R) between variables $X_{i}$ and $Y_{i}$ (on a Scale between -1 and +1 )}

$$
R=\frac{\sum_{i=1}^{n}(\text { Xi-Xmean })(\text { Yi-Ymean })}{\sqrt{\sum_{i=1}^{n}(X i-X \text { Xean })^{2} \sum_{i=1}^{n}(\text { Yi-Ymean })^{2}}}
$$

\section{Coefficient of Determination $=R^{2}$ (on Scale between 0 and 1)}

Note that Eq. (11) and the squaring of Eq. (12) to derive $\mathrm{R}^{2}$ provide the same results as the correlation coefficient and coefficient of determination functions in Excel (i.e. CORREL and RSQ), respectively.

\section{Results}

\section{Relationships between Variables in the DSDG Dataset}

The data variable ranges and means of the 10,000 cases included in the DSGC data set are listed in Table 2. Of these cases, 3620 were calculated (Eq.(4)) to result in a stable grid condition $\left(S t b_{\text {in }}\right.$ negative) and 6380 were associated with an unstable grid condition $\left(S t b_{\text {in }}\right.$ negative).

The LHS sampling with no specific dependencies applied among the variables (dependent or independent) leads to very low correlations among the independent variables. This is revealed by the correlation matrix for the full DSGC dataset (Table 3 ) in which correlation coefficient values ( $\mathrm{R}$ from Eq. (11)) are displayed.

Between almost all the independent variables $\mathrm{R}$ is $<0.02$. The exceptions are between the electricity production (P1) and consumption variables (P2 to $\mathrm{P} 4$ ) for which $\mathrm{R}$ is $\sim 0.58\left(\mathrm{R}^{2} \sim 0.33\right)$. The simulation model allowed $\mathrm{P} 1$ to $\mathrm{P} 4$ to vary independently but applied the constraint that for each case $\mathrm{P} 1=\sum(\mathrm{P} 1$ to $\mathrm{P} 4)$. This explains the observed correlations between $\mathrm{P} 1$ and the electricity consumption variables $\left(\mathrm{R}^{2} \sim 0.33\right.$ for each), despite there being no correlations among P2 to P4 variables $(\mathrm{R}<0.013)$. Dependent variable $S t b_{\text {in }}$ shows no correlation with P1 to P4, suggesting that grid stability is independent of electricity volumes flowing through the grid with the range constraints applied (Table 2). However, $S t b_{\text {in }}$ does show minor positive correlations (Fig. 2) individually with the Tau1 to Tau4 and G1 to $\mathrm{G} 4$ variables ( $\mathrm{R}$ in the range 0.28 to $0.30 ; \mathrm{R}^{2} 0.08$ to 0.09 ), indicating that for the simulation assumptions grid stability is more influenced by the commercially-related independent variables. Considered on a compound basis for the consuming participants (Table 4), taking the average of Tau 2 to Tau4 and the average of G2 to G4, somewhat more significant correlations (Fig. 3) are displayed with $S t b_{\text {in }}$ ( $\mathrm{R}$ in the range 0.56 to $0.58 ; \mathrm{R}^{2} 0.31$ to 0.33 ), despite there being no correlation between the sum of $\mathrm{P} 2$ to P4 and $S t b_{i n}$.

The correlation relationships between the input variables and the dependent variable also vary subtly for the cases that result in grid stability versus the cases that result in grid instability (Table 5).

For the segment of the dataset that results in grid instability the correlation between the average G2 to G4 and $S t b_{i n}$ is much higher $(\mathrm{R}=0.55)$ than for the segment resulting in grid stability $(\mathrm{R}=0.24)$. This is also the case, but with lower $\mathrm{R}$ values, for G1 to G4 individually (Table 5) and the correlations between Tau1 to Tau4 and the average of Tau2 toTau4 and $S t b_{i n}$. The correlation coefficient between the average of Tau2 to Tau4 and $S t b_{\text {in }}$ for the unstable grid segment $(\mathrm{R}=$ $0.35)$ is slightly greater than that for the stable grid segment $(R=0.30)$, but both of these show lower $R$ values between these variables than for the full dataset $(\mathrm{R}=0.56)$ (Table 5). These relationships are influenced by the subtly variations in the poor correlations between the Tau and $\mathrm{G}$ variables in the stable and unstable grid segments of the dataset (Table 6). Whereas, Tau and G show essentially no correlations among them for the full dataset, this is not the case for the stable and unstable grid segments considered individually.

For the complete dataset $\mathrm{R}$ for the $\mathrm{G}$ versus Tau variables is in the E-04 to E-07 range (no correlation). For the unstable segment of the grid $\mathrm{R}$ for the $\mathrm{G}$ versus Tau variables is in the E-02 to E-03 range (very poor correlation). For the stable segment of the grid $\mathrm{R}$ for the $\mathrm{G}$ versus Tau variables is in 
Table 2 Value ranges and means for the DSGC simulated dataset [24] for which the grid stability metric $\mathrm{Stb}_{\text {in }}$ is the dependent variable and the other twelve variables are the independent variables

DSGC Simulated Dataset

$(10,000$ records

\begin{tabular}{llll} 
) & & \\
Variable & Min & Max & Mean \\
Tau1 & 0.5008 & 9.9995 & 5.2500 \\
Tau2 & 0.5001 & 9.9998 & 5.2500 \\
Tau3 & 0.5008 & 9.9995 & 5.2500 \\
Tau4 & 0.5505 & 9.9994 & 5.2500 \\
P1 & 1.5826 & 5.8644 & 3.7500 \\
P2 & -1.9999 & -0.5001 & -1.2500 \\
P3 & -1.9999 & -0.5001 & -1.2500 \\
P4 & -1.9999 & -0.5000 & -1.2500 \\
G1 & 0.0500 & 0.9999 & 0.5250 \\
G2 & 0.0501 & 0.9999 & 0.5250 \\
G3 & 0.0501 & 1.0000 & 0.5250 \\
G4 & 0.0500 & 0.9999 & 0.5250 \\
Stb & -0.0808 & 0.1094 & 0.0157 \\
\hline
\end{tabular}

For 3620 records $\mathrm{Stb}_{\text {in }}<=0$ (grid stable); for 6380 records $\mathrm{Stb}_{\text {in }}>0$ (grid unstable)

the E-02 to E-06 range (very poor correlation to no correlation), but interestingly, the correlations for this segment between G1 and Tau1, G2 and Tau2, G3 and Tau 3, G4 and Tau4 are all in the $\mathrm{E}-02$ range. These very subtle distinctions may be an artefact of the variable sampling technique. As will be shown, these subtle very poor correlations do have some significance when it comes to predicting the dependent variable for this dataset by machine learning methods.
Applying TOB Learning Network to the Simulated DSGC Dataset

The TOB learning network is applied initially to the complete DSGC dataset (12 independent variables) with a series of sensitivity cases run with different sized tuning and testing subsets. The results of ten sensitivity cases are displayed in Tables 7 to 11 .

The ten cases (Table 7) are configured with a range of data records distributed between the tuning (left side), testing (right side) and training (extreme right-hand column) subsets. For most of the cases, the accuracy of four alternative $S t b_{\text {in }}$ predictions are displayed for both the tuning subset and with those solutions ( $Q$ value and variable weights) applied to the testing subset. These alternative prediction are: the TOB stage 1 prediction, the TOB Stage 2 prediction applying the coded memetic firefly optimizer; the TOB Stage 2 prediction applying the Excel Solver's GRG optimizer; and, the Excel Solver's evolutionary optimizer. For the cases with tuning subsets larger than 100 data records the Solver optimizers have not been applied due to the time required to enter cell formulas into a spreadsheet.

For the testing subset the performance of TOB stage 1 is quite consistent (Table 6) for all the sensitivity cases run (RMSE from 0.01608 to $0.01705 s^{-2} ; \mathrm{R}^{2}$ from 0.82451 to $0.87269)$. It demonstrates that by matching with the closest ten records in the training subset based on the sums of the weighted squared errors ( $\left.\sum \mathrm{SSE}\right)$ for all twelve independent variables with equal weights applied TOB stage 1 can achieve good prediction accuracy (Fig. 4). It is clear from this cross plot of predicted versus measured, that the accuracy achieved improves as becomes more positive. This is due to the

Table 3 Correlation matrix displaying correlation coefficient $(\mathrm{R})$ for the DSGC simulated dataset [24] for which the grid stability metric $\mathrm{Stb}_{\text {in }}$ is

the dependent variable and the other twelve variables are the independent variables

Correlation Matrix for the Variables in the DSGC Dataset (10,000 records)

\begin{tabular}{|c|c|c|c|c|c|c|c|c|c|c|c|c|c|}
\hline $\mathrm{R}$ & Tau1 & Tau2 & Tau3 & Tau4 & P1 & $\mathrm{P} 2$ & P3 & P4 & G1 & G2 & G3 & G4 & $\mathrm{Stb}_{\text {in }}$ \\
\hline Tau1 & 1.000 & 0.016 & -0.006 & -0.017 & 0.027 & -0.015 & -0.016 & -0.016 & 0.011 & 0.015 & -0.001 & 0.005 & 0.276 \\
\hline Tau2 & 0.016 & 1.000 & 0.014 & -0.002 & -0.005 & 0.007 & 0.008 & -0.006 & -0.002 & 0.015 & 0.017 & -0.012 & 0.291 \\
\hline Tau3 & -0.006 & 0.014 & 1.000 & 0.004 & 0.017 & -0.003 & -0.009 & -0.018 & -0.012 & 0.008 & 0.015 & -0.011 & 0.281 \\
\hline Tau4 & -0.017 & -0.002 & 0.004 & 1.000 & -0.003 & 0.011 & 0.006 & -0.011 & -0.004 & 0.008 & 0.003 & 0.000 & 0.279 \\
\hline $\mathrm{P} 1$ & 0.027 & -0.005 & 0.017 & -0.003 & 1.000 & -0.573 & -0.585 & -0.579 & 0.001 & 0.015 & 0.001 & -0.015 & 0.010 \\
\hline P2 & -0.015 & 0.007 & -0.003 & 0.011 & -0.573 & 1.000 & 0.002 & -0.007 & 0.016 & -0.018 & 0.008 & 0.020 & 0.006 \\
\hline P3 & -0.016 & 0.008 & -0.009 & 0.006 & -0.585 & 0.002 & 1.000 & 0.013 & -0.003 & -0.012 & -0.006 & -0.010 & -0.003 \\
\hline P4 & -0.016 & -0.006 & -0.018 & -0.011 & -0.579 & -0.007 & 0.013 & 1.000 & -0.014 & 0.003 & -0.004 & 0.018 & -0.021 \\
\hline G1 & 0.011 & -0.002 & -0.012 & -0.004 & 0.001 & 0.016 & -0.003 & -0.014 & 1.000 & 0.008 & -0.006 & 0.012 & 0.283 \\
\hline G2 & 0.015 & 0.015 & 0.008 & 0.008 & 0.015 & -0.018 & -0.012 & 0.003 & 0.008 & 1.000 & -0.013 & -0.015 & 0.294 \\
\hline G3 & -0.001 & 0.017 & 0.015 & 0.003 & 0.001 & 0.008 & -0.006 & -0.004 & -0.006 & -0.013 & 1.000 & 0.007 & 0.308 \\
\hline G4 & 0.005 & -0.012 & -0.011 & 0.000 & -0.015 & 0.020 & -0.010 & 0.018 & 0.012 & -0.015 & 0.007 & 1.000 & 0.279 \\
\hline $\mathrm{Stb}_{\text {in }}$ & 0.276 & 0.291 & 0.281 & 0.279 & 0.010 & 0.006 & -0.003 & -0.021 & 0.283 & 0.294 & 0.308 & 0.279 & 1.000 \\
\hline
\end{tabular}


Fig. 2 Tau2 versus $\mathrm{Stb}_{\text {in }}$ for one of the independent variables that displays a minor correlation with the dependent variable (grid stability metric) for the DSGC simulated dataset [24]

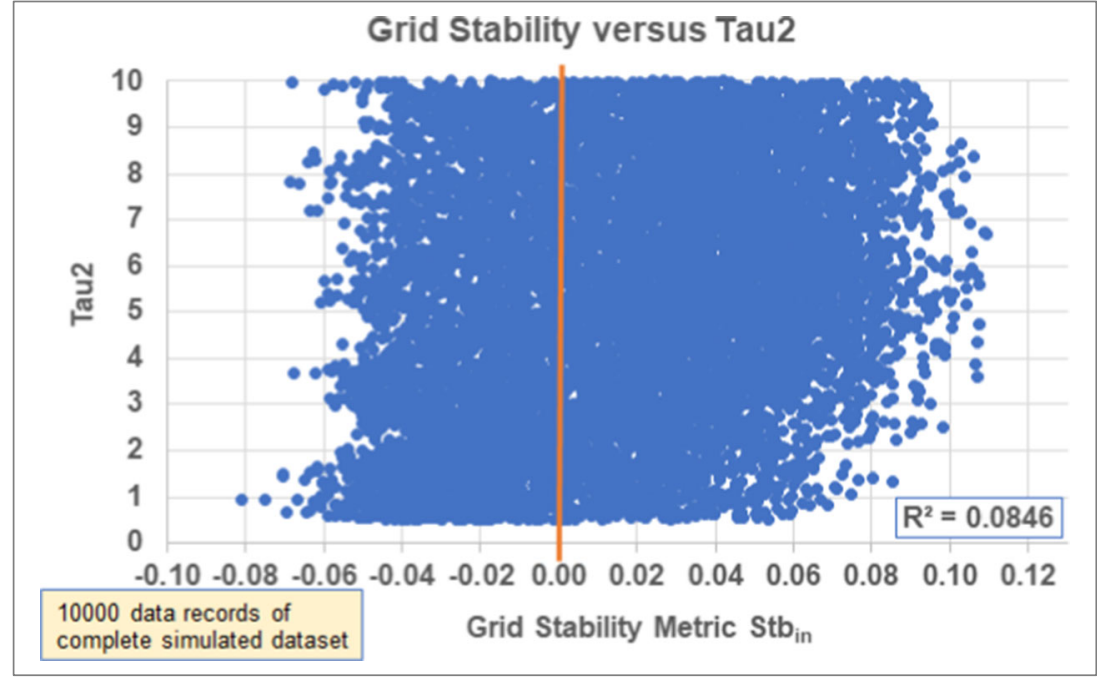

correlation differences described between the grid-stable and grid-unstable segments of the dataset.

Comparing the prediction performance of TOB stage 1 and TOB stage 2 for the testing subsets is a useful way to determine whether the dataset is being overfitted by the optimized solution derived from the tuning subset. If the prediction accuracy is better for TOB stage 1 results than TOB stage 2 results that is a sure indicator that the data is being overfitted. It generally means that the tuning subset size (number of data records) is too small. This is the case for Cases \#4 (76 data records in the tuning subset) and Case\#5 (51 data records in the tuning subset) (Tables 7 and 9). For Cases \#1, \#2,\#3,\#7, and \#8 (all with 100 data records in the tuning subset) this comparison is not so clear cut. For Cases\#1, \#2 and \#3 the TOB stage 2 prediction for the testing subset are better than the TOB stage 1 predictions but for Cases\#7 and \#8 they are slightly worse. This suggests that $\sim 100$ data records in the tuning subset is close to the minimum required to avoid over fitting.

For Cases \#6, \#9 and \#10 the prediction performance of TOB stage 2 is clearly superior (but only by a small amount)

Table 4 Correlation matrix displaying correlation coefficient (R) for the DSGC simulated dataset [24] for three compound independent variables and the grid stability metric $\mathrm{Stb}_{\text {in }}$

Correlation Matrix for Compound Variables in DSGC Dataset $(10,000$ records)

\begin{tabular}{lcccc}
\hline R & $\begin{array}{l}\text { Average } \\
\text { Tau2 to Tau4 }\end{array}$ & $\begin{array}{l}\text { Sum } \\
\text { P2 to P4 }\end{array}$ & $\begin{array}{l}\text { Average } \\
\text { G2 to G4 }\end{array}$ & Stb $_{\text {in }}$ \\
\hline Average Tau2 to Tau 4 & 1.000 & -0.018 & 0.014 & 0.562 \\
$\begin{array}{l}\text { Sum } \\
\text { P2 to P4 }\end{array}$ & -0.018 & 1.000 & -0.001 & -0.10 \\
$\begin{array}{l}\text { Average } \\
\text { G2 to G4 }\end{array}$ & 0.014 & -0.001 & 1.000 & 0.583 \\
Stb & 0.562 & -0.010 & 0.583 & 1.000 \\
\hline
\end{tabular}

than TOB Stage 1. This indicates that with tuning subsets of $>=147$ records the TOB Stage 2 optimization is picking out valid relationships in the dataset. For those three datasets the TOB Stage 2 predictions vary within a relative narrow range (RMSE from 0.01252 to $0.01651 s^{-2} ; \mathrm{R}^{2}$ from 0.83912 to 0.86090 ). The improvement in prediction accuracy achieved by TOB Stage 2 is very small in comparison with TOB Stage 1 , which is to be expected due to the almost negligible correlations that exist between the independent variable for the complete dataset (Table 3).

Tables 7 and 8 highlight that although it is possible to achieve quite impressive prediction performance for a small tuning subset ( $<=100$ data records), when applied to an independent testing subset that prediction performance is not replicated (e.g. Cases \#4 and \#5) across a range of statistical performance indicators (Eq. (5) to Eq. (12)). It is the results for the independent testing subsets (Table 9) on which these optimized solutions derived should be judged not the prediction results for the tuning subsets (Table 8 ). The TOB Stage 2 solutions from all ten cases applied to the large testing case of 916 data records summarizes that performance (Table 10). The first row in that table displays the TOB Stage 1 results which are useful to use to benchmark the TOB Stage 2 performance. It is apparent that only the cases relating to the last three rows of Table 10 have achieved demonstrable improvements on the predictions easily derived by TOB stage 1 , and those improvement unimpressively small.

The main limitation on the TOB Stage 2 prediction performance for this dataset is the lack of correlation between the individual independent variable, a feature imposed on the dataset by the random uniform LHC sampling. Clearly it does not make a huge difference using constant squared-error weights of 0.5 (TOB Stage 1) or squared-error weights allowed to vary between 0 and 1 (TOB Stage 2) applied to the independent variables of this dataset when deriving predictions (Table 10). However, it is revealing to look at the 
Fig. 3 Average Tau2 to Tau4 versus $\mathrm{Stb}_{\text {in }}$ for one of the compound independent variables that displays a moderate correlation with the dependent variable (grid stability metric) for the DSGC simulated dataset [24]

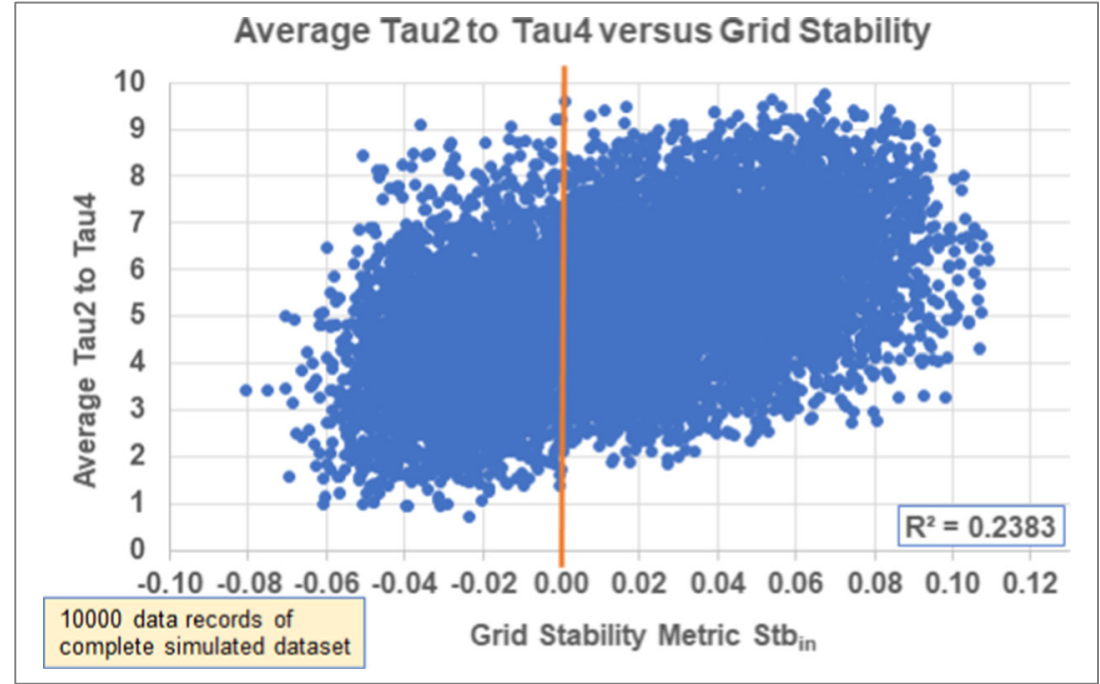

variable squared-error weights that are selected by the TOB Stage 2 optimum solutions (Table 11).

What stands out for the final few rows in Table 11 is that the optimum TOB Stage 2 solutions select high weight for Tau variables, in some instances also the $\mathrm{G}$ variables, but apply near-zero squared-error weights to the $\mathrm{P}$ variables. This is consistent with the more significant correlations observed between Tau 1 to 4 and $S t b_{i n}$ and between G 1 to 4 and $S t b_{i n}$, but negligible correlations between P 1 to 4 and $S t b_{\text {in }}$ (Table 3). There are even stronger correlations between average Tau 2 to

Table 5 Correlation coefficient (R) for key segments of the DSGC simulated dataset [24] between key independent variables and the grid stability metric $\mathrm{Stb}_{\text {in }}$

\begin{tabular}{llll} 
Correlation Coefficients for Key Segments of the DSGC Dataset \\
\hline & Full Dataset & $\begin{array}{l}\text { Grid Table } \\
<=1\end{array}$ & Grid Unstable \\
& & 360 & 6380 \\
Cases & 10,000 & 3620 & Stb $_{\text {in }}$ \\
R & Stb $_{\text {in }}$ & Stb $_{\text {in }}$ & 0.175 \\
Tau1 & 0.276 & 0.105 & 0.176 \\
Tau2 & 0.291 & 0.140 & 0.173 \\
Tau3 & 0.281 & 0.126 & 0.167 \\
Tau4 & 0.279 & 0.117 & 0.354 \\
Average Tau2 to Tau4 & 0.562 & 0.304 & 0.005 \\
P1 & 0.010 & 0.001 & 0.000 \\
P2 & 0.006 & 0.008 & -0.005 \\
P3 & -0.003 & -0.006 & -0.004 \\
P4 & -0.021 & -0.003 & -0.005 \\
Sum P2 to P4 & -0.010 & -0.001 & 0.243 \\
G1 & 0.283 & 0.156 & 0.248 \\
G2 & 0.294 & 0.108 & 0.255 \\
G3 & 0.308 & 0.106 & 0.236 \\
G4 & 0.279 & 0.108 & 0.552 \\
Average G2 to G4 & 0.583 & 0.243 & \\
\hline
\end{tabular}

Tau4 and average G2 to G4 and $S t b_{\text {in }}$ (Table 5). This indicates that the optimizers applied as part of TOB stage 2 are exploiting indirectly these relationships among the dataset variables.

Based on the information provided in Table 11, it is possible to explore ways to improve the optimized prediction performance of the TOB learning network. As the P2 to P4 variables contribute little to the optimized solutions an alternative worth considering is to combine variables $\mathrm{P} 2$ to $\mathrm{P} 4$ into a single compound variable (Sum P2 to P4) and replace variable

Table 6 Correlation coefficient $(\mathrm{R})$ for $\mathrm{G}$ versus Tau for the complete DSGC simulated dataset [24] and for the stable grid and unstable grid segments of the simulation considered individually

Subtle Correlations Between Tau and G in Segments of the DSGC Simulated Dataset

Full Dataset (10,000 records)

$\begin{array}{lllll}\text { R } & \text { Tau1 } & \text { Tau2 } & \text { Tau3 } & \text { Tau4 } \\ \text { G1 } & 1.11 \mathrm{E}-04 & 3.04 \mathrm{E}-06 & 1.35 \mathrm{E}-04 & 1.72 \mathrm{E}-05 \\ \text { G2 } & 2.36 \mathrm{E}-0.04 & 2.37 \mathrm{E}-04 & 5.89 \mathrm{E}-05 & 7.11 \mathrm{E}-05 \\ \text { G3 } & 1.63 \mathrm{E}-06 & 2.73 \mathrm{E}-04 & 2.16 \mathrm{E}-04 & 1.06 \mathrm{E}-05 \\ \text { G4 } & 3.02 \mathrm{E}-05 & 1.38 \mathrm{E}-04 & 1.32 \mathrm{E}-04 & 2.41 \mathrm{E}-07 \\ \text { Grid } & \text { Table (3620 records) } & & & \\ \text { R } & \text { Tau1 } & \text { Tau2 } & \text { Tau3 } & \text { Tau4 } \\ \text { G1 } & 4.85 \mathrm{E}-02 & 6.86 \mathrm{E}-07 & 3.01 \mathrm{E}-04 & 3.77 \mathrm{E}-04 \\ \text { G2 } & 1.64 \mathrm{E}-06 & 4.16 \mathrm{E}-02 & 1.65 \mathrm{E}-04 & 1.63 \mathrm{E}-05 \\ \text { G3 } & 3.51 \mathrm{E}-05 & 5.88 \mathrm{E}-04 & 5.22 \mathrm{E}-02 & 1.45 \mathrm{E}-03 \\ \text { G4 } & 1.70 \mathrm{E}-06 & 6.97 \mathrm{E}-04 & 8.66 \mathrm{E}-06 & 5.34 \mathrm{E}-02 \\ \text { Grid Unstable }(6380 \text { records) } & & \\ \text { R } & \text { Tau1 } & \text { Tau2 } & \text { Tau3 } & \text { Tau4 } \\ \text { G1 } & 6.07 \mathrm{E}-03 & 7.51 \mathrm{E}-03 & 7.91 \mathrm{E}-03 & 5.75 \mathrm{E}-03 \\ \text { G2 } & 3.71 \mathrm{E}-03 & 3.77 \mathrm{E}-03 & 4.52 \mathrm{E}-03 & 5.99 \mathrm{E}-03 \\ \text { G3 } & 8.51 \mathrm{E}-03 & 7.08 \mathrm{E}-03 & 4.88 \mathrm{E}-03 & 4.36 \mathrm{E}-03 \\ \text { G4 } & 5.55 \mathrm{E}-03 & 8.15 \mathrm{E}-03 & 1.10 \mathrm{E}-02 & 3.60 \mathrm{E}-03\end{array}$




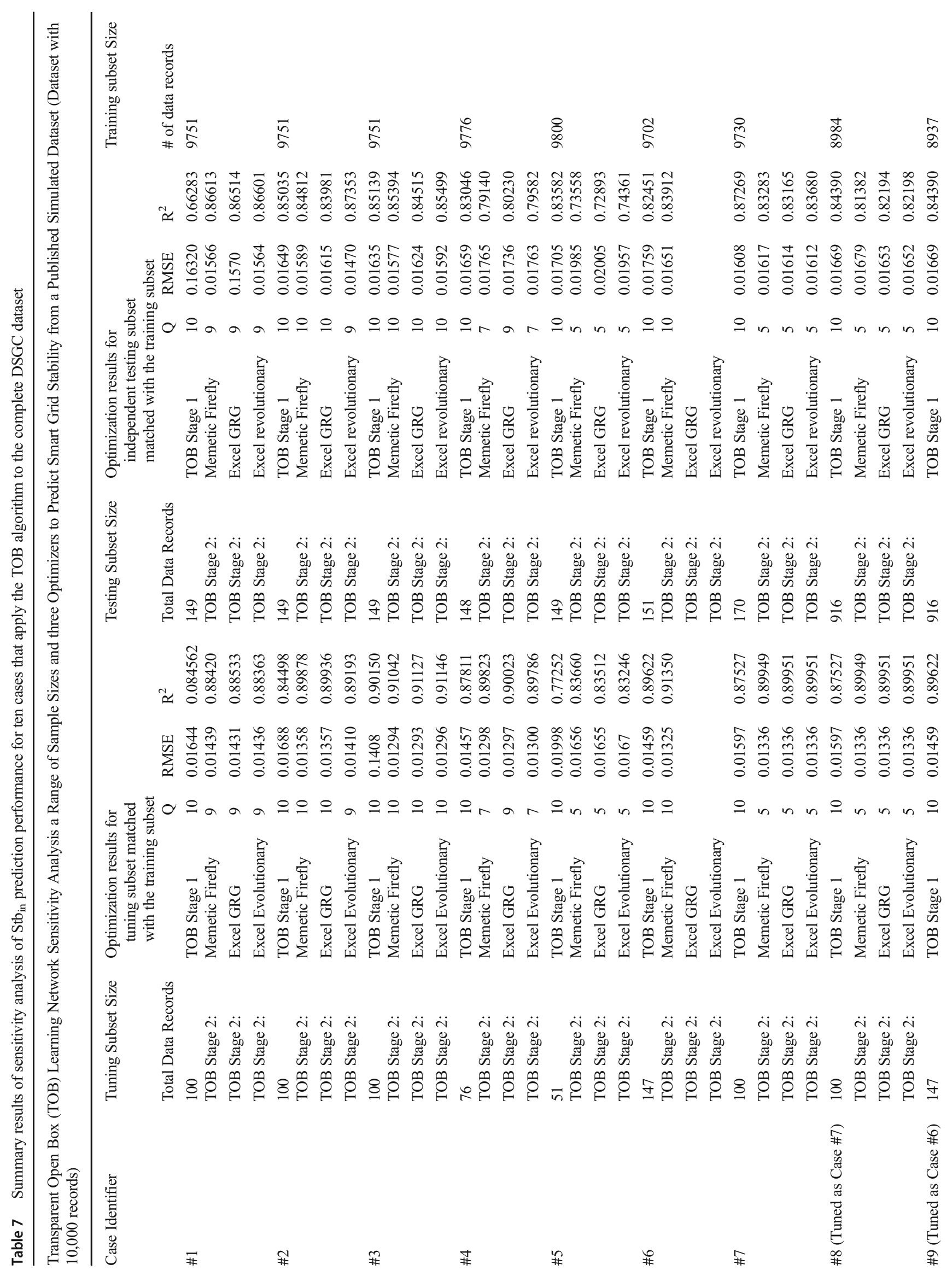


P3 in the dataset with that compound metric. This opens the opportunity to replace variable $\mathrm{P} 2$ with the compound variable "average Tau 2 to Tau 4" and to replace variable P4 with the compound variable "average G2 to G4". This adjusted dataset still retains the same number of variables but the compound variables make the independent variable components less random (Table 12).

Case\#12 evaluates this adapted dataset with the TOB algorithm using a tuning set of 147 data records and a testing subset of 151 data records and does demonstrate a more accurate prediction performance (Table 13 and Fig. 5). Note that the optimum TOB Stage 2 solution applies high weights to the Tau 1 to 4 variables and the "average Tau2 to Tau4" and "average G2 to G4" compound variables to achieve its improved predictions.

A supplementary data file providing the adjusted dataset divided into the training, tuning and testing subsets used for Case \#12 is available (Appendix 2).

\section{Discussion}

Effectively managing and controlling demand response in decentralized electricity grids is challenging. This is because the consumption / production behaviour of grid participants is driven by price signals issued and responded to on the scale of seconds. The stability of such grids is influenced by a number of variables related to the physical attributes and complexity of the grid. Key influencing variables are the cost-sensitive status of the participants (price elasticity, abbreviated as $\gamma$ or $\mathrm{G})$ and the ability of the participants to react to price signals on scale of a few seconds (abbreviated as $\tau$ or Tau).

The Decentral Smart Grid Control (DSGC) decentralized grid management and pricing system [6] offers the potential to achieve effective demand response on the time scales required by linking electricity price to grid frequency. A simulation of grid stability for DSGC applied to a four-node star grid (Fig. 1), based on its key controlling independent variables, including $\mathrm{G}$ and Tau, has been shown to effectively explore the solution space of that decentralised grid design [24]. The scenario simulated in that dataset involves four grid participants with a very low level of dependency between their independent variables. It is the simulated scenario evaluated by the TOB algorithm in this study.

Arzamasov et al. [24] have effectively used the CART decision-tree algorithm to data mine and provide some insight to this dataset. That decision tree analysis correctly classified design points with a prediction accuracy for grid stability $\left(\mathrm{R}^{2}\right.$ $\sim 0.80$ ). The main contributions of this study applying TOB, combined with statistical analysis are:

- to provide grid stability predictions in a novel way by avoiding correlations between the variables. 
Table 8 Seven statistical measures of $\mathrm{Stb}_{\text {in }}$ prediction accuracy for the optimum TOB solution derived for the tuning subsets of ten sensitivity cases applied to the complete DSGC dataset

Tuning Sunset TOB Stage 2 Performance Accuracy Metrics for the Optimum Case Found by the Memetic Firefly Optimizer

\begin{tabular}{|c|c|c|c|c|c|c|c|}
\hline Case Identifier & RMSE Mj/kg & MSE $\mathrm{Mj} / \mathrm{kg}$ & $\mathrm{APD} \%$ & AAPD $\%$ & $\mathrm{SD}$ & $\mathrm{R}$ & $\mathrm{R}^{2}$ \\
\hline Case\#1 & 0.01431 & 0.00020 & 30.09286 & 91.04497 & 0.01428 & 0.94092 & 0.88533 \\
\hline Case\#2 & 0.01358 & 0.00018 & -38.33784 & 130.70179 & 0.01361 & 0.94804 & 0.89878 \\
\hline Case\#3 & 0.01294 & 0.00017 & 1.57592 & 62.80097 & 0.01292 & 0.95416 & 0.91042 \\
\hline Case\#4 & 0.1298 & 0.00017 & 77.09508 & 114.17727 & 0.01305 & 0.94775 & 0.89823 \\
\hline Case\#5 & 0.01656 & 0.00027 & 26.86381 & 71.01395 & 0.01662 & 0.91466 & 0.83660 \\
\hline Case\#6 & 0.01325 & 0.00018 & 13.60574 & 85.97295 & 0.01313 & 0.9557 & 0.91350 \\
\hline Case\#7 & 0.04336 & 0.00018 & 44.74258 & 84.05263 & 0.01343 & 0.94842 & 0.89949 \\
\hline Case\#8 & 0.01225 & 0.00018 & 44.74258 & 84.05263 & 0.01343 & 0.94842 & 0.89949 \\
\hline Case\#9 & 0.01325 & 0.00018 & 13.60574 & 85.97295 & 0.01313 & 0.95577 & 0.91350 \\
\hline Case\#10 & 0.01434 & 0.00021 & 8.74797 & 111.75690 & 0.01428 & 0.94184 & 0.88706 \\
\hline
\end{tabular}

Case\#10 is highlighted as this is considered to be the most statistically valid of the cases run

- to achieves improved grid stability prediction accuracy; $\mathrm{R}^{2}$ varies between about 0.86 and 0.90 , depending upon the input metrics selected.

- to reveal complementary information with respect to $S t b_{\text {in }}$ predictions regarding the relative contribution of the input variables to prediction accuracy and the prediction accuracy achieved for specific segments of the calculated grid stability distribution are revealed (e.g., Table 5).

However, the simulated four-star node dataset evaluated here represents a very simply decentralized grid configuration. To more comprehensively evaluate and better define the dynamic grid stability performance of DSGC, there is clearly the need for future studies to conduct further simulations on: 1) larger decentralized grids, (e.g. 5 to 10 participants, and $>10$ participants); 2) grids involving multiple prosumers; and, 3 ) different grid architectures (e.g. circular and multi-branched configurations).

The assumed behavioural relationships between the participants in that simulated grid (i.e., complete independence with no inherent correlations each sampled with random uniform distributions) is also idealized. The twelve independent variables (Tau1 to Tau4, P1 to P4, G1 to G4) are sampled as random uniform distributions with the only constraints applied being minimum and maximum limits on each variable (Table 3 ) and $\mathrm{P} 1+\mathrm{P} 2+\mathrm{P} 3+\mathrm{P} 4=0$ for each simulation case. This approach is very effective at fully exploring the feasible solution space with the 10,000 cases evaluated, as it was designed to do. However, in real decentralized grids it is likely that there would be some slightly more significant correlations, albeit complex and non-linear ones, between the behaviours of the participants and their responses to price

Table 9 Seven statistical measures of $\mathrm{Stb}_{\text {in }}$ prediction accuracy for the optimum TOB solution derived for the testing subsets of ten sensitivity cases applied to the complete DSGC dataset

Testing Subset TOB Stage 2 Performance Accuracy Metrics for the Optimum Case Found by the Memetic Firefly Optimizer

\begin{tabular}{|c|c|c|c|c|c|c|c|}
\hline Case Identifier & RMSE Mj/kg & MSE MJ/kg & APD $\%$ & AAPD $\%$ & SD & $\mathrm{R}$ & $\mathrm{R}$ \\
\hline Case \#1 & 0.01570 & 0.00025 & 76.36281 & 120.56600 & 0.01552 & 0.93013 & 0.86514 \\
\hline Case \#2 & 0.01589 & 0.00025 & 53.73404 & 120.51189 & 0.01564 & 0.92093 & 0.84812 \\
\hline Case \#3 & 0.01577 & 0.00025 & 13.30152 & 79.60956 & 0.01561 & 0.92409 & 0.85394 \\
\hline Case \#4 & 0.01765 & 0.00031 & -37.31452 & 122.01271 & 0.01753 & 0.88961 & 0.79140 \\
\hline Case \#5 & 0.01985 & 0.00039 & 45.64318 & 102.02251 & 0.01973 & 0.85766 & 0.73558 \\
\hline Case \#6 & 0.01651 & 0.00027 & 71.94960 & 109.62548 & 0.01649 & 0.91603 & 0.83912 \\
\hline Case \#7 & 0.01617 & 0.00026 & 40.03990 & 126.23447 & 0.01603 & 0.91260 & 0.83283 \\
\hline Case \#8 & 0.1679 & 0.00028 & 58.88503 & 129.26977 & 0.01663 & 0.90212 & 0.81382 \\
\hline Case \#9 & 0.01552 & 0.00024 & 28.19072 & 99.61400 & 0.01530 & 0.92785 & 0.86090 \\
\hline Case \#10 & 0.01568 & 0.00025 & 29.22230 & 101.80000 & 0.01541 & 0.92676 & 0.85888 \\
\hline
\end{tabular}

Case\#10 is highlighted as this is considered to be the most statistically valid of the cases run 
Table 10 Performance of prediction accuracy for the optimized solutions (variable weights and Q values) for the ten sensitivity cases applied to a testing subset of 916 records the complete DSGC dataset

TOB Stage 2 performance Accuracy Metrics for the Optimum Solutions found by the Memetic Firefly Optimizer Applied 916-data-record testing Subset and Compared to TOB Stage 1 Prediction Performance for that dataset

\begin{tabular}{|c|c|c|c|c|c|c|c|c|c|}
\hline Case Identifier & $\begin{array}{l}\text { Tuning Subset } \\
\text { \#Data records }\end{array}$ & RMSE Mj/kg & MSE MJ/kg & APD $\%$ & AAPD $\%$ & SD & $\mathrm{R}$ & $\mathrm{R}^{2}$ & $\begin{array}{l}\text { Performance Relative } \\
\text { to TOB Stage } 1\end{array}$ \\
\hline TOB Stage 1 & N/A & 0.01669 & 0.00028 & 45.26816 & 118.12046 & 0.01634 & 0.91864 & 0.84390 & Benchmark \\
\hline case\#5 & 51 & 0.01906 & 0.00036 & 53.88974 & 148.87810 & 0.01882 & 0.86861 & 0.75449 & \multirow{3}{*}{$\begin{array}{l}\text { Generate a less } \\
\text { accurate prediction } \\
\text { than TOB Stage } 1\end{array}$} \\
\hline Case\#4 & 76 & 0.01789 & 0.00032 & 61.48925 & 151.66466 & 0.01756 & 0.89240 & 0.79638 & \\
\hline Case\#7 \& \#8 & 100 & 0.01679 & 0.00028 & 58.88503 & 129.26977 & 0.01663 & 0.90212 & 0.81382 & \\
\hline Case\#1 & 100 & 0.01619 & 0.00026 & 30.57080 & 106.51584 & 0.01589 & 0.91992 & 0.84626 & \multirow{2}{*}{$\begin{array}{l}\text { Match but do not } \\
\text { improve upon } \\
\text { TOB stage } 1\end{array}$} \\
\hline Case\#2 & 100 & 0.01604 & 0.00026 & 23.74633 & 101.91158 & 0.01581 & 0.92105 & 0.84834 & \\
\hline Case\#3 & 100 & 0.01578 & 0.00025 & 32.47710 & 103.67095 & 0.01556 & 0.92426 & 0.85426 & \multirow{3}{*}{$\begin{array}{l}\text { Slightly improve upon } \\
\text { TOB Stage } 1 \mathrm{~s}\end{array}$} \\
\hline Case \#6 \& \#9 & 147 & 0.01552 & 0.00024 & 28.19072 & 99.61400 & 0.01530 & 0.92785 & 0.86090 & \\
\hline case\#10 & 200 & 0.01568 & 0.00025 & 29.22230 & 101.80000 & 0.01541 & 0.92676 & 0.85888 & \\
\hline
\end{tabular}

fluctuations, i.e., relationships between their reaction times to price changes (Tau); their price elasticities $(\mathrm{G})$, and the changes they would make in the quantities of electricity they would produce / consume (P). Such correlations should significantly limit / constrain the likelihood of certain cases materializing within the feasible solution spaces defined. More centralized distributions of the Tau, G and P are also more likely in real grids than the uniform distributions used to explore the feasible solution space in the simulated case studied. The presence of more extensive correlations and centralized distributions is likely to improve the $S t b_{\text {in }}$ prediction accuracies of machine learning algorithms, including TOB in predicting grid stability.

Fig. 4 Predicted versus actual $\mathrm{Stb}_{\text {in }}$ for sensitivity case \#10 for TOB Stage one applied to an independently selected 200record testing subset extracted from the entire DSCG dataset
The transparent attributes of the TOB algorithm, enabling it to provide forensic analysis of each prediction it makes [44], could also be exploited to reveal the significance of the subtle relationships between the DSGC variables of more complex grid configurations.

The balance between data transparency and data security / protection is an important issue in the design of decentralized power grids. For market confidence and credible price discovery it is essential that the metrics influencing electricity price are transparently recorded and reported. On the other hand, to inhibit potential cyberattacks it is important that grid attributes (mechanical and/or commercial classifiers) that could be exploited to precipitate grid instability cannot be externally

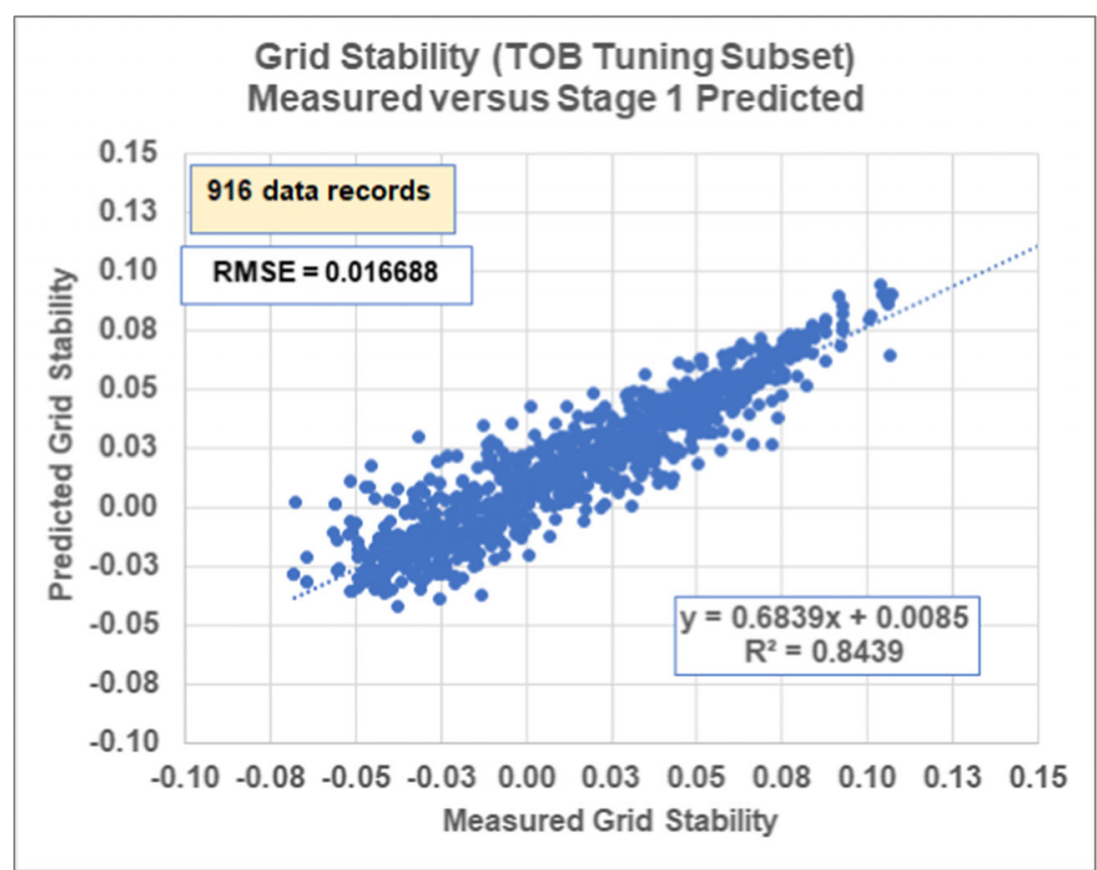




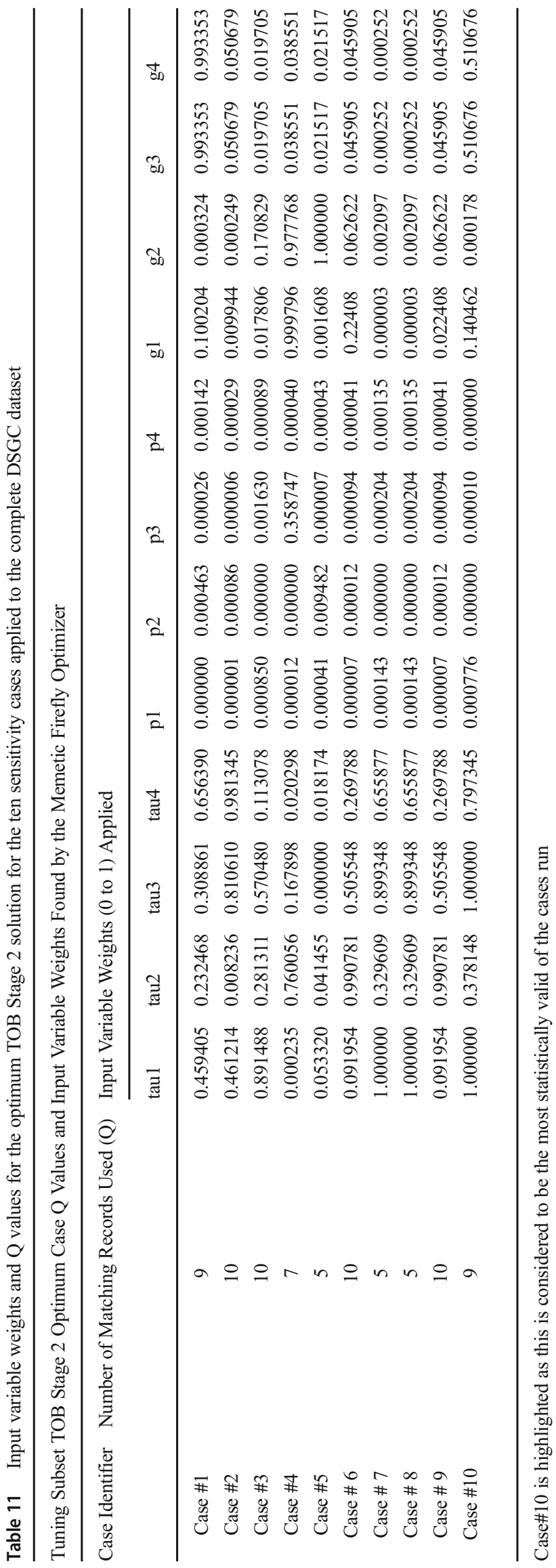

manipulated. Note that whereas the system operator should be able to calculate quite specifically grid stability conditions from a suite of relatively complex differential equations (such as Eq.(4)), other grid participants (major producers, consumers and prosumers) would likely not have access to the necessary information to do so. However, the grid participants and third parties (without access to all the mechanical and commercial data for the DSGC) are likely, for commercial and competitive reasons, to be interested in price prediction, discovery, forecasting and economic modelling. Indeed, reliable price discovery is essential for market confidence in such grids. Consequently, grid participants and price discovery agencies need to be able to predict price relationships governed by grid stability and grid frequency mechanisms from estimates of the key variables involved. These variables would likely include grid frequency and G, P, and Tau distributions (or other defined and lesscommercially-revealing but cost-sensitive metrics defined for grid participants) for each grid participant. Correlation-free, data-matching, machine-learning algorithms, such as TOB, applied to datasets established with such variables for specific decentralized grids managed by DSGC principles, have the potential to provide the necessary prediction accuracy and transparency required by system operators, grid participants and third parties without compromising grid security. Further studies of more complex decentralized grid configurations are required to verify that potential.

\section{Conclusions}

This study applies the transparent open box (TOB) machinelearning algorithm to evaluate a published virtual simulation of decentralized smart grid performance with the objective of predicting the stability of that grid accurately and transparently. The TOB model is applied to a Decentral Smart Grid Control (DSGC) dataset that simulates electricity price linked to grid frequency. Key input variables that influence grid stability in that model are the power produced or consumed by the grid participants $(\mathrm{P})$ the cost sensitivity of the grid participants $(\mathrm{G})$ and the ability of grid participants to react to electricity price signals (Tau). The model evaluated involves four grid participants with low levels of interdependency between either their P, G or Tau values.

Applying the transparent open box (TOB) learning algorithm to a published DSGC simulated dataset demonstrates how its two-stage prediction process, coupled with detailed statistical analysis of the relationships between the variables, can provide further insight to the DSGC functionality. TOB achieves a grid stability prediction accuracy root mean squared error (RMSE) of about $0.016 \mathrm{~s}^{-2}$ and a coefficient of determination $\left(\mathrm{R}^{2}\right)$ of about 0.85 for the dataset just involving the poorly correlated $\mathrm{P}, \mathrm{G}$ and Tau variables of the four participants. However, when compound variables (average 
Table 12 Correlation matrix displaying correlation coefficient $(\mathrm{R})$ for an adaption to the DSGC simulated dataset [24] for which the grid stability metric $\mathrm{Stb}_{\text {in }}$ is the dependent variable but with three compound metrics replacing P2 to P4 variables of the original dataset as shown in Table 3

Correlation Matrix for the Variables in the DSGC Dataset (10,000 records) with three Compound Variables substituted for Variables P2 to P4 of the Original Dataset

\begin{tabular}{|c|c|c|c|c|c|c|c|c|c|c|c|c|c|}
\hline $\mathrm{R}$ & Tau1 & Tau2 & Tau3 & Tau4 & $\mathrm{P} 1$ & $\begin{array}{l}\text { Avg Tau2 } \\
\text { to Tau4 }\end{array}$ & $\sum \mathrm{P} 2$ to $\mathrm{P} 4$ & Avg G2 To G4 & G1 & G2 & G3 & G4 & $\mathrm{Stb}_{\text {in }}$ \\
\hline Tau1 & 1.000 & 0.016 & -0.006 & -0.017 & 0.027 & 0.495 & -0.027 & 0.015 & 0.011 & 0.015 & -0.001 & 0.005 & 0.276 \\
\hline Tau2 & 0.016 & 1.000 & 0.014 & -0.002 & -0.005 & 0.513 & 0.005 & 0.009 & -0.002 & 0.015 & 0.017 & -0.012 & 0.291 \\
\hline Tau3 & -0.006 & 0.014 & 1.000 & 0.004 & 0.017 & 0.505 & -0.017 & 0.000 & -0.012 & 0.008 & 0.015 & -0.011 & 0.281 \\
\hline Tau4 & -0.017 & -0.002 & 0.004 & 1.000 & -0.003 & 0.491 & 0.003 & 0.004 & -0.004 & 0.008 & 0.003 & 0.000 & 0.279 \\
\hline $\mathrm{P} 1$ & 0.027 & -0.005 & 0.017 & -0.003 & 1.000 & 0.018 & -1.000 & 0.001 & 0.001 & 0.015 & 0.001 & -0.015 & 0.010 \\
\hline Avg Tau2 & 0.495 & 0.513 & 0.505 & 0.491 & 0.018 & 1.000 & -0.018 & 0.014 & -0.003 & 0.023 & 0.017 & -0.009 & 0.562 \\
\hline to Tau4 & -0.027 & 0.005 & -0.017 & 0.003 & -1.000 & -0.018 & 1.000 & -0.001 & -0.001 & -0.015 & -0.001 & 0.015 & -0.010 \\
\hline$\sum \mathrm{P} 2$ to $\mathrm{P} 4$ & 0.015 & 0.009 & 0.000 & 0.004 & 0.001 & 0.014 & -0.001 & 1.000 & 0.508 & 0.491 & 0.495 & 0.503 & 0.583 \\
\hline Avg G2 & 0.011 & -0.002 & -0.012 & -0.004 & 0.001 & -0.003 & -0.001 & 0.508 & 1.000 & 0.008 & -0.006 & 0.012 & 0.283 \\
\hline To G4 & 0.015 & 0.015 & 0.008 & 0.008 & 0.015 & 0.023 & -0.015 & 0.491 & 0.008 & 1.000 & -0.013 & -0.015 & 0.294 \\
\hline G1 & -0.001 & 0.017 & 0.015 & 0.003 & 0.001 & 0.017 & -0.001 & 0.495 & -0.006 & -0.013 & 1.000 & 0.007 & 0.308 \\
\hline G2 & 0.005 & -0.012 & -0.011 & 0.000 & -0.015 & -0.009 & 0.015 & 0.503 & 0.012 & -0.015 & 0.007 & 1.000 & 0.279 \\
\hline \multicolumn{14}{|l|}{ G3 } \\
\hline \multicolumn{14}{|l|}{ G4 } \\
\hline $\mathrm{Stb}_{\text {in }}$ & 0.276 & 0.291 & 0.281 & 0.279 & 0.010 & 0.562 & -0.010 & 0.583 & 0.283 & 0.294 & 0.308 & 0.279 & 1.000 \\
\hline
\end{tabular}

Table 13 Improved optimum solutions established by TOB Stage 1 and Stage 2 algorithms applied to the adapted DSGC dataset including compound independent variables

Case \#12 DSGC Dataset (10,000 records) (tuning subset 147;testing subset 151)

\begin{tabular}{lll}
\hline optimum Solution & TOB Stage 1 & TOB Stage 2 \\
\hline RMSE & 0.00806 & 0.00745 \\
MSE & $6.4954 \mathrm{E}-05$ & $5.55558 \mathrm{E}-05$ \\
APD\% & -9.676 & -7.765 \\
AAPD\% & 16.840 & 15.443 \\
SD & 0.00799 & 0.00745 \\
R & 0.9416 & 0.9501 \\
R $^{2}$ & 0.8866 & 0.9026 \\
Q & 10 & 10 \\
Weights & & \\
Tau1 & 0.5 & $9.842 \mathrm{E}-01$ \\
tau2 & 0.5 & $3.456 \mathrm{E}-01 \mathrm{~S}$ \\
Tau3 & 0.5 & $2.117 \mathrm{E}-01$ \\
Tau4 & 0.5 & $3.455 \mathrm{E}-01$ \\
P1 & 0.5 & $0.000 \mathrm{E}+00$ \\
Avg Tau2 to Tau4 & 0.5 & $9.917 \mathrm{E}-01$ \\
EP2 TO P4 & 0.5 & $5.640 \mathrm{E}-06$ \\
Avg G2 to G4 & 0.5 & $7.553 \mathrm{E}-02$ \\
G1 & 0.5 & $8.701 \mathrm{E}-04$ \\
G2 & 0.5 & $5.877 \mathrm{E}-02$ \\
G3 & 0.5 & $1.470 \mathrm{E}-04$ \\
G4 & 0.5 & $1.470 \mathrm{E}-04$ \\
\hline
\end{tabular}

Tau and average $\mathrm{G}$ for the three consuming participants) are introduced as independent variables, the TOB algorithm is able to substantially improve it grid stability predictions (RMSE $\sim 0.0075 \mathrm{~s}^{-2} ; \mathrm{R}^{2} \sim 0.90$ ). A key finding of this study is therefore that assessing the stability of decentralized systems by including input variables with certain levels of dependency can improve predictions of grid stability.

Other novel and significant findings of this study are: 1) the magnitudes of the variable weights applied by TOB Stage 2 indicate that it is able to exploit the underlying relationships (both high and low correlations) that exist among the independent variables without actually relying on such correlations to derive its predictions. 2) TOB's ability to forensically interrogate the contributions made by specific dataset records to each TOB prediction (Stage 1 or Stage 2) could be used effectively to enhance transparency in the electricity pricing controls of a decentralized DSGC grid. 3) The provision of transparent information, such as that provided by the TOB prediction model, to all grid participants and third parties (such as price discovery agents) with which to estimate electricity price trends would likely enhance market confidence and price discovery for DSGC systems without compromising grid security and stability.

More complex decentralized grids would likely involve much more complex relationships, displayed variable degrees of dependency between their input variables and grid stability. This should enhance the ability of the TOB data-matching, machine-learning algorithm's ability to achieve highly accurate predictions of grid stability exploiting such dependent input variables. Future studies are required to simulate more complex grid configurations using higher numbers of 
Fig. 5 Improved $\mathrm{Stb}_{\text {in }}$ prediction performance by TOB Stage 2 applied to the adapted DSGC dataset (Case \#12)

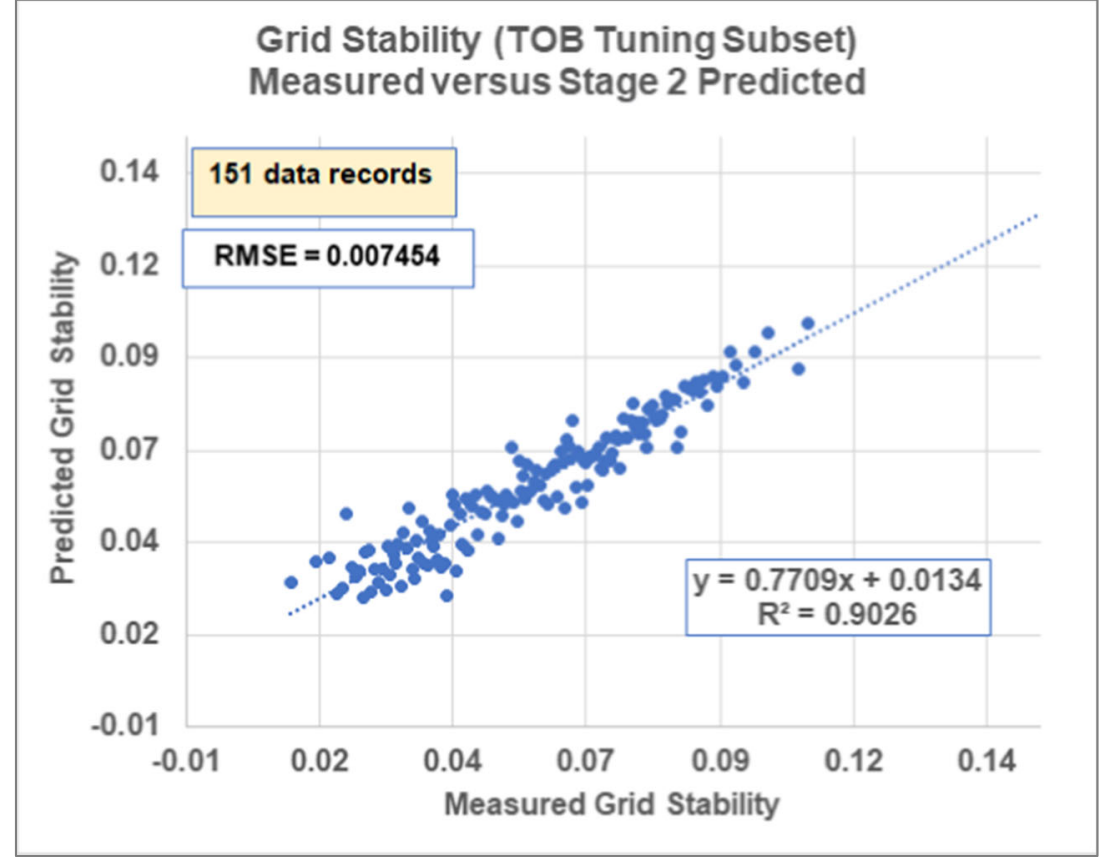

participants. Applying TOB analysis to transparently and accurately predict the stability of more complex grid is also required to confirm the methods prediction and data mining capabilities.

\section{Compliance with Ethical Standards}

Conflict of Interest The author declares no conflicts of interest related to the topics addressed in this study.

Nomenclature $\alpha_{j}$, Damping constant related to the power dynamics of the grid; $A P D$, Average percent deviation; $A A P D$, Absolute average percent deviation; $c_{1}$, Proportionality factor; $D S G C$, Decentral smart grid control; $f$, Fractional contribution to VSE; $\gamma_{j}=$, A coefficient proportional to cost sensitivity /price elasticity (also referred to as $\mathrm{G}) ; j$, Grid participant identifier ( $j=1$ is designated the producer; $j=2$ to 4 are designated to the consumers); $K$, Coupling strength between grid participants $j$ and $k$; LHS, Latin hypercube simulation; $M h$, Metaheuristic; MSE, Mean square error; $n, n^{\text {th }}$ variable; $N$, Number of independent variables; $p_{j}$, is the electricity price for grid participant $j ; \hat{P}_{j}$, is the power produced or consumed by grid participant $j$ at electricity price $p_{j .} ; p_{\omega}$, is the electricity price when no net change in electricity is flowing through the grid; $P D i$, Percent deviation; $q$, One of the top-ranking data records used in predictions; $Q$, The maximum number of top-ranking data records used in predictions $(2<=\mathrm{Q}<=10) ; R$, Correlation coefficient; $R^{2}$, Coefficient of determination; RMSE, Root mean square error; $\mathrm{s}^{-2}$, Per second per second; $S D$, Standard deviation; $S t b_{i n}$, Dynamic grid stability metric simulated; $\theta_{j}$ and $\theta_{k}$, Are rotor angles for grid participants $j$ and $k$ at a specific point in time $t ; t$, Refers to a specific point in time; $T_{j}$, is the time interval used to define electricity prices; $\tau_{j}$, is a grid participants reaction time (in seconds) also referred to as Tau; te, Testing subset; $t r$, Training subset; $t u$, Tuning subset; TOB, Transparent open box machine learning algorithm; UCI, University of California, School of Information and Computer Science; VBA, Visual basic for applications; VSE, Variable squared error; $W n$, Weight applied to the VSE of variable $n ; X_{i}$, the $i^{\text {th }}$ data record in the data set being predicted

\section{Appendix 1 Outline of TOB Learning Network Method}

The steps and mathematical basic for the optimized nearestneighbour, transparent open-box (TOB) algorithm [1, 43] are summarized here.

\section{General Basis of TOB Algorithm}

The TOB algorithm has attributes that relate it to locallyweighted learning methods [45] following lazy-learning principles [46] that built upon nearest-neighbour matching [47, 48] that also provide valuable transparency [49]. Nearest neighbour and k-learning algorithms are successfully applied to solve many pattern recognition challenges [50-53]. Nearest-neighbour-prediction algorithms often attempt to linearize non-smooth dataset locally around small groups of data records [54]. The TOB algorithm uses squared errors between the independent variables to establish close matches between records in a dataset to derive its stage 1 predictions of dependent variables. It then applies an optimizer with variable weightings applied to the squared errors of closely matching records to derive its Stage 2 predictions.

\section{TOB Stage 1 (Data Matching to Provide an Initial Prediction)}

Step 1: Assemble a 2-D array [number of variables $(N+1)$, number of data records $(M)] . N$ independent variables plus the dependent variable to be predicted.

Step 2: Sort data records in value order of the dependent variable. 
Step 3: Generate statistical summary for the dataset including maximum and minimum of each variable (e.g. Table 2).

Step 4: Use maximum and minimum values to normalize all data variables to a range of -1 to +1 by applying Eq. A1.

$X_{i} *=2 *\left[\left(X_{i}-X \min \right) /(X \max -X \min )\right]-1$

Where:

$X_{i} \quad$ the $i^{t h}$ data record for $X$ of $N+1$ variables.

$X$ min minimum of variable $X$ for the entire dataset.

$X \max$ maximum of variable $X$ for all data records.

$X_{i}^{*} \quad$ normalized value for the $i^{t h}$ record for $X$ of $N+1$

variables.

Step 5: Verify that the complete dataset is appropriately normalized and sorted in order of the dependent variable, which makes visual inspection of the data records easier.

Step 6: Divide the complete dataset into three subsets: a large training subset and smaller tuning and independent testing subset. Trial-and -error sensitivity analysis is required to establish the optimum sizes for the tuning and training subset to provide meaningful data and avoid overfitting. Comparing the prediction performances of TOB stages 1 and 2 for the testing subset is a useful indicator of overfitting [55]. A tuning subset size is selected such that the TOB Stage 2 TOB predictions for the testing subset consistently and reliably outperform the stage 1 predictions for the testing subset.

The requirement for separate tuning and training subsets is to enable the optimizer to tune the weights applied to the independent variables in the records of the training subset to provide better predictions for a representative, but relatively small, tuning subset records. This reduces computational time.

Step 7: Calculate squared errors of the independent variables (VSE) for each of $J$ tuning-subset data records versus the $K$ training-subset data records using Eq. A2:

$\operatorname{VSE}(X)_{j k}=\left[X_{k}(t r)-X_{j}(t u)\right]^{2}$

Where:

$X_{k}(t r) \quad$ variable $X$ value for the $k^{\text {th }}$ training-subset record.

$X_{j}(t u) \quad$ variable $X$ for the $j^{\text {th }}$ tuning-subset record.

$\operatorname{VSE}(X)_{j k} \quad$ variable-squared error (VSE) for variable $X$ of the $j^{\text {th }}$ tuning-subset record versus variable $X$ of the $k^{\text {th }}$ training-subset record. $\sum V S E_{j k}$ computes the weighted sum of the computed VSE values applying Eq. A3:

$\sum V S E_{j k}=\sum_{n=1}^{n=N+1} \operatorname{VSE}(X n)_{j k} *(W n)$

Where:

$S E(X n)_{j k} \quad$ the variable-squared error (VSE) for variable $X n$ for the $j^{\text {th }}$ tuning-subset record versus the $k^{\text {th }}$ training-subset record.

$\sum V S E_{j k} \quad$ sum of variable-squared errors (VSE) for the $N+1$ variables (including the dependent variable) for the $j^{\text {th }}$ tuning-subset record versus the $k^{\text {th }}$ data training-subset record.

$W n=$ weights $(0<W n<=1)$ applied to the calculated VSE for all variables involved in the prediction (i.e., $N+1$ ). Each weight is set to a constant value (e.g. 0.5 or 1.0) in TOB stage 1. This means that TOB Stage 1 establishes the high-ranking data record matches initially without any biases introduced by weights applied to the squared errors of the independent variables.

Step 8: For TOB Stage 1Establish the top-ten matching records $(Q=10)$ in the training subset for each record in the tuning subset, i.e. those data records with the lowest calculated $\sum$ VSE.

Step 9: The contribution fraction applied to the top-Q-(Q = 10)-ranked, matching records is calculated applying Eq. A4, Eq. A5 and Eq. A6. The computed contribution fraction depends upon the relative magnitudes of the $\sum V S E$ values for each training-subset record versus the $j^{\text {th }}$ tuning-subset record.

$$
f_{j q}=\sum V S E_{j q} /\left[\sum_{r=1}^{r=Q} \sum V S E_{j r}\right]
$$

Where:

$q$ the $q^{\text {th }}$ of Q top-ranking training-subset records from the training subset for the $j^{\text {th }}$ tuning subset record.

$r$ the $r^{\text {th }}$ of Q top-ranking training-subset records from the training subset for the $j^{\text {th }}$ tuning subset record.

$f_{q}$ the contribution fraction calculated for the $q^{\text {th }}$ of Q topranking records for the $j^{\text {th }}$ tuning subset record.

Eq.(A5) imposes a key constraint that normalizes the $f_{q}$ values to sum to 1 . Q always equals 10 for TOB stage 1 .

$\sum_{q=1}^{q=Q} f_{q}=1$ 
The data record in the training subset with the highestranking match (i.e., the one with the lowest $\sum V S E_{j k}$ value) then makes the greatest contribution to the prediction of the dependent variable associated with the $j^{\text {th }}$ tuning-subset record. This is achieved by applying $\left(1-f_{q}\right)$ multipliers in Eq. A6

$$
\left(X_{N+1}\right)_{j}^{\text {predicted }}=\sum_{q=1}^{q=Q}\left[\left(X_{N+1}\right)_{q} *\left(1-f_{q}\right)\right]
$$

Where:

$\left(X_{N+1}\right)_{q}$ Dependent variable for the $q^{\text {th }}$ training-subset record (i.e., one of $\mathrm{Q}$ best-matching records).

$\left(X_{N+1}\right)_{j}^{\text {predicted }}$ TOB-stage-one predicted-dependentvariable value for the $j^{\text {th }}$ tuning-subset record.

TOB Stage 1 calculations provide a provisional prediction based upon a fixed number of matching records $(\mathrm{Q}=10)$ and equal weights $(W n)$ applied to the squared errors between the variables. This prediction is further refined in TOB stage 2 by applying an optimizer and allowing $Q$ and $W n$ to vary within specified limits.

Step 10: The accuracy of the TOB Stage 1 predictions are assessed in terms of the statistical accuracy metrics defined in the main text (Eq. 5 to Eq. 12) with closest attention paid to root mean squared error (RMSE), used as the objective function of the optimizer in TOB Stage 2.

\section{TOB Stage 2 (Optimizing the Weights and Number of Matching Records)}

Step 11: An optimizer is applied to minimize RMSE for the collective predictions calculated for the tuning subset (i.e., the $J$ tuning-subset data records). Q and $W_{n}$ are allowed to vary (unlike in TOB Stage 1):

The $N$ input-variable weights $\left(W_{n}\right)$ applied to the squared errors are allowed to vary $(0<W n<=1)$.

The optimizer is allowed to vary $\mathrm{Q}(2<=\mathrm{Q}$ $<=10$ ) defining how many of the best-matching records should be used for the prediction calculations (Eqs. A4, A5 and A6).

Eq. A3 is re-evaluated in the optimization calculations by varying $W_{n}$ across its constrained range. TOB stage-2 $\sum V S E_{j q}$ values are recomputed with Eq. A4 for different $Q$ values $(2<\mathrm{Q}<=10)$ in each iteration of the optimizer.

For tuning subsets with less that about 100 data records, the Generalized Reduced Gradient (GRG) non-linear and evolutionary optimization algorithm options of the standard "Solver" optimizer available in Microsoft Excel spreadsheets [56] can be conveniently employed for TOB
Stage 2 optimization. These are readily applied using visual basic for application (VBA) code combined with calculations for Eqs. A3 to A6 setup as cell formulas in an Excel spreadsheet. For mid-sized and large datasets, such as the DSGC dataset evaluated here, with tuning subsets containing more than 100 data records it is more convenient to fully code a customized optimizer without the nee to enter the calculations as Excel cell formulas. TOB can be configured in a fully coded manner in any programming language (.e.g., Octave, Matlab, Python, VBA etc.). For the DSGC dataset a customized memetic firefly optimizer [43] coded in VBA is applied and the results compared with Excel Solver results for some of the smaller tuning subsets evaluated (Table 7).

The firefly optimizer was developed $[57,58]$ with computational analogies made to the behaviour of fireflies. Fireflies progressively move towards brighter individuals in a swarm incorporating some random components to their positional adjustments. The firefly algorithm adjusts the position of each member of a population based on specific rules related to a calculated "brightness" metric that is proportional to how close their objective function value is to the lowest value achieved so far [59]. The greatest brightness is assigned to the highest-performing solution and the lowest brightness to the poorest-performing solution $[60,61]$.

The memetic firefly algorithm, customized specifically to the requirements of the TOB learning network, involves six integrated memes or metaheuristics $(\mathrm{Mh})$ [43]. These memes improve its convergence speed, compared to the standalone firefly optimizer, to the global minimum. They enhance its ability to resist becoming trapped it sub-optimal local minima. These memes operate on each of the multiple iterations applied to a progressively modified population of potential solutions:

Mh1: Creates some solutions generated randomly;

Mh2: Creates some solutions applying the firefly algorithm;

Mh3: Creates some solutions by making minor adjustments to several selected variables ( $W n$ or $Q$ ) of existing highranking solutions;

Mh4: Creates some solutions by adjusting a single variable weights to very low $(\sim 0)$ or very high values $(\sim 1)$ for existing high-ranking solutions;

Mh5: Creates some solutions by mutating one randomly selected variable ( $W n$ or $Q$ ) in existing solutions of various rank;

Mh6: Creates some solutions by modifying one variable (Wn or $Q$ ) by alternating large and small amounts in each of the top-ten ranked existing solutions.

The effectiveness of each meme in contributing to a solution is monitored by a metaheuristic profiling technique $[62$, 63], a graphical representation of which is illustrated by Fig. 6 for an optimization solution derived for Case\#12. 
Fig. 6 Metaheuristic profile (Wood, $2016 \mathrm{a}, \mathrm{b}$ ) for iterations 1 to 110 of the memetic firefly optimizer as it progresses towards a minimum RMSE solution for sensitivity Case \#12. The top-ten solutions found by each meme identified for each iteration of the algorithm

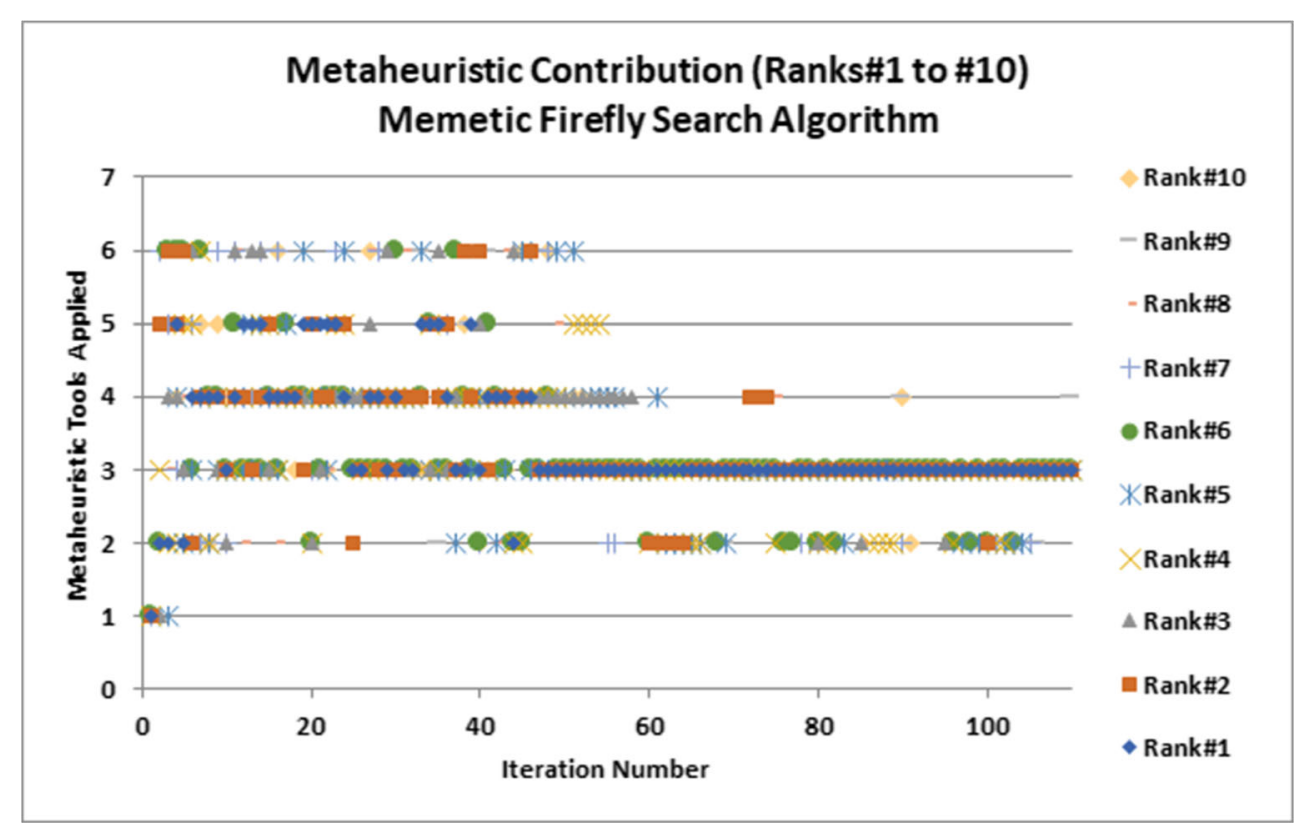

Figure 6 indicates that memes Mh2 to Mh6 are all frequently involved in providing a top-ten ranking solutions for the memetic firefly optimizer up to iteration \#50 or so. On the other hand, random solutions only contribute to the top-ten solutions in the first few iterations Beyond iteration 60 the top-ten solutions are dominated by $\mathrm{Mh} 2$ and Mh3. Such profiling verifies that all the memes in the memetic algorithm are contributing to the performance of the optimizer as a whole. The optimizer collects the details (Wn and Q values) for all the solutions it evaluates. This is an advantage over the Solver optimizers that just provide details of the best solution found.

Step 12: The accuracy of the TOB Stage 2 predictions for the tuning subset are assessed in terms of the statistical accuracy metrics defined in the main text (Eq. 5 to Eq. 12) and compared with the statistical accuracy achieved by TOB Stage 1 for the tuning subset. It is often revealing to perform sensitivity analysis in step 12 by optimizing with different fixed values of $\mathrm{Q}$ (i.e. $\mathrm{Q}=2$ to 10 ) as it helps to assess underfitting or overfitting issues associated with the data set being evaluated.

Step 13: Step 13. Compute TOB Stage 1 and TOB Stage 2 predictions for all the data records in the independent testing-subset by applying the optimum values established for $W_{n}$ and $Q$ in step 11 for the tuning subset. Assessing the full set of statistical accuracy measures (Eq. 5 to 12) and comparing them for stage- 1 and stage- 2 predictions is important. If stage- 2 predictions are less accurate than stage- 1 predictions for the testing subset it is an indication that the optimization of the tuning set is overfitting the data and probably a larger tuning subset is required. For most datasets TOB Stage 2 predictions can improve on the prediction accuracy achieved by TOB Stage 1. It is often useful in Stage 13 to audit the intermediate calculation for individual records in the tuning subset steps to reveal which variables are having the greatest impact on the TOB predictions. The TOB algorithm readily allows forensic investigation of all the calculations involved in the predictions of each data record [44].

Step 14: Compare the results provided by the TOB algorithm and associated statistical analysis with empirical calculations and/or other machine-learning and data-mining algorithms and, if available, empirical correlations. Such comparisons can be used to complement the insights provided to the dataset (e.g., decision tree analysis of the DSGC dataset) and/or benchmark the prediction performance.

\section{Appendix 2 Supplementary Data File}

A supplementary Excel data file is provided splitting the DSGC simulated dataset adapted with three compound variables for Case\#12 into training, tuning and testing subsets.

\section{References}

1. Wood DA (2018a) A transparent open-box learning network provides insight to complex systems and a performance benchmark for more-opaque machine learning algorithms. Adv Geo-Energy Res 2(2):148-162 
2. Ackermann T, Andersson G, Soder L (2001) Distributed generation: a definition. Electr Power Syst Res 57(3):195-204

3. Bremdal BA (2014) The impact of prosumers in a smart grid-based energy market. Meter Int 2:71-73

4. Troester E (2009) New German grid codes for connecting PV systems to the medium voltage power grid. In, 2nd international workshop on concentrating photovoltaic power plants: optical design, production, grid connection, march edition: 9-10

5. Milan P, Wachter M, Peinke J (2013) Turbulent character of wind energy. Phys Rev Lett 110(13):138701. https://doi.org/10.1103/ PhysRevLett.110.138701

6. Schäfer B, Matthiae M, Timme M, Witthaut D (2015) Decentral smart grid control. New J Phys 17(1):15002

7. Palensky P, Dietrich D (2011) Demand side management: demand response, intelligent energy systems, and smart loads. IEEE Trans Indust Inform 7(3):381-388. https://doi.org/10.1109/TII.2011. 2158841

8. Albadi MH, El-Saadany EF (2008) A summary of demand response in electricity markets. Electr Power Syst Res 78(11):19891996. https://doi.org/10.1016/j.epsr.2008.04.002

9. Butler D (2007) Energy efficiency super savers: meters to manage the future. Nature 445(7128):586-588. https://doi.org/10.1038/ $445586 \mathrm{a}$

10. Chauhan RK, Phurailatpam C, Rajpurohit BS, Gonzalez-Longatt FM, Singh SN (2017) Demand-side management system for autonomous DC microgrid for building. Technol Econ Smart Grids Sustain Energy 2:4, 11 pages-11. https://doi.org/10.1007/s40866017-0020-y

11. Fouladfar MH, Loni A, Tookanlou MB, Marzband M, Godina R, Al-Sumaiti A, Pouresmaeil E (2019) The impact of demand response programs on reducing the emissions and cost of a neighborhood home microgrid. Appl Sci 9:2097, 27 pages. https://doi.org/ 10.3390/app9102097

12. Javadi M, Marzband M, Akorede MF, Godina R, Al-Sumaita AS, Pouresmaeil (2018) A centralized smart decision-making hierarchical interactive architecture for multiple home microgrids in retail electricity market. Energies 11:3144, 22 pages. https://doi.org/10. 3390/en11113144

13. Siddiqui AN, Thomas MS (2016) Techno-Economic Evaluation of Regulation Service from SEVs in Smart MG System. Technol Econ Smart Grids Sustain Energ 1:15-10. https://doi.org/10.1007/ s40866-016-0016-Z

14. Ericsson GN (2010) Cyber security and power system communication - essential parts of a smart grid infrastructure. IEEE Trans Power Deliv 25(3):1501-1507. https://doi.org/10.1109/TPWRD. 2010.2046654

15. Fang X, Misra S, Xue G, Yang D (2012) Smart grids - the new and improved power grid: a survey. Commun Surv Tutor IEEE 14(4): 944-980. https://doi.org/10.1109/SURV.2011.101911.00087

16. Lu S, Samaan N, Diao R, Elizondo M, Jin C, Mayhorn E, Zhang Y, Kirkham H (2011) Centralized and decentralized control for demand response. In IEEE Innovative Smart Grid Technologies (ISGT), January 17-19, 2011, Anaheim, California, USA. 8 pages. doi:https://doi.org/10.1109/ISGT.2011.5759191

17. Schweppe FC (1982) Frequency adaptive, power-energy re-scheduler, February 23 1982. US Patent 4:317-049

18. Short JA, Infield DG, Freris LL (2007) Stabilization of grid frequency through dynamic demand control. IEEE Trans Power Syst 22(3):1284-1293. https://doi.org/10.1109/TPWRS.2007.901489

19. Timme M, Kocarev L, Witthaut D (2015) Focus on networks, energy and the economy. New J Phys 17(11):110201. https://doi.org/ $10.1088 / 1367-2630 / 17 / 11 / 110201$

20. Roos C (2017) Decentral smart grid control: how different EV control strategies affect the dynamical grid stability. Thesis. University of Utrecht. 38 pages
21. Strbac G (2008) Demand side management: Benefits and challenges. Energy Policy 36(12):4419-4426

22. Schäfer B, Grabow C, Auer S, Kurths J, Witthaut D, Timme M (2016) Taming instabilities in power grid networks by decentralized control. Eur Phys J: Spec Topics 225(3):569-582

23. Schäfer B, Witthaut D, Timme M (2018) How Decentral Smart Grid Control Limits Non-Gaussian Power Grid Frequency Fluctuations. 2018 IEEE conference on control technology and applications (CCTA). doi: https://doi.org/10.1109/CCTA.2018. 8511400

24. Arzamasov V, Bohm K, Jochem P (2018) Towards concise models of grid stability. IEEE international conference on communications, control, and computing Technologies for Smart Grids (SmartGridComm) 2018 (section V-A)

25. UCI Machine Learning Repository (2018) Electrical Grid Stability Simulated. 10000 point / 14 attribute data set deposited by V Arzamasov in November 2018. https://archive.ics.uci.edu/ml/ datasets/Electrical+Grid+Stability+Simulated+Data+ (Accessed 15 January 2019)

26. Glavic M, Fonteneau R, Ernst D (2017) Reinforcement learning for electric power system decision and control: past considerations and perspectives. IFAC PapersOnLine 50(1):6918-6927

27. Jasmin EA, Ahamed TPI, Raj VPJ (2011) Reinforcement learning approaches to economic dispatch problem. Int. journal Elec. Power Ener Syst 33:836-845

28. Li FD, Wu H, He Y, Chen X (2012) Optimal control in micro grid using multi-agent reinforcement learning. ISA Trans 51:743-751

29. Mocanu E (2017) Machine learning applied to smart grids. Thesis, Eindhoven: Technische Universiteit Eindhoven, 166 pages

30. Kofinas P, Dounis AI, Vouros GA (2018) Fuzzy Q-learning for multi-agent decentralized energy management in microgrids. Appl Energy 219:53-67

31. Menke JH, Schafer F, Braun M (2018) Performing a Virtual Field Test of a New Monitoring Method for Smart Power Grids. 2018 IEEE international conference on communications, control, and computing Technologies for Smart Grids (SmartGridComm). doi: https://doi.org/10.1109/SmartGridComm.2018.8587551

32. Amraee T, Ranjbar S (2013) Transient instability prediction using decision tree technique. IEEE Trans Power Syst 28(3):3028-3037. https://doi.org/10.1109/TPWRS.2013.2238684

33. Moulin LS, Alves da Silva AP, El-Sharkawi MA, Marks RJ (2004) Support vector Machines for Transient Stability Analysis of largescale power systems. IEEE Trans Power Syst 19(2):818-825. https://doi.org/10.1109/TPWRS.2004.826018

34. LeCun Z, Bengio Y, Hinton G (2015) Deep learning. Nature 521: 436- 444

35. Sogabe T, Malla DB, Takayama S, Sakamoto K, Yamaguchi K, Singh TP, Sogabe M (2018) Smart Grid Optimization by Deep Reinforcement Learning over Discrete and Continuous Action Space. The 32nd Annual Conference of the Japanese Society for Artificial Intelligence 4 pages

36. Kaygusuz C, Babun L, Aksu H, Uluagac AS (2018) Detection of compromised smart grid devices with machine learning and convolution techniques. IEEE international conference on communications (ICC 2018) 6 pages. doi:https://doi.org/10.1109/ICC.2018. 8423022

37. Kosek AM (2016) Contextual anomaly detection for cyber-physical security in smart grids based on an artificial neural network model. In, joint workshop on cyber- physical security and resilience in smart grids (CPSR-SG), April 2016, 1-6

38. Nishikawa T, Motter AE (2015) Comparative analysis of existing models for power-grid synchronization. New J Phys 17(1):15012

39. Schmietendorf K, Peinke J, Friedrich R, Kamps O (2014) Selforganized synchronization and voltage stability in networks of synchronous machines. Eur Phys J Spec Topics 2592:2577-2592 
40. Johnson RT, Montgomery DC, Kennedy KS (2012) Hybrid spacefilling designs for computer experiments. Front Stat Qual Control 10:287-301

41. Stein M (2014) Large sample properties of simulations using Latin hypercube sampling. Technometrics 29(January):37-41

42. Breiman L (1984) Classification and regression trees. Routledge, New York. 368 pages (e-edition 2017). https://doi.org/10.1201/ 9781315139470

43. Wood DA (2018b) Thermal maturity and burial history modelling of shale is enhanced by use of Arrhenius time-temperature index and memetic optimizer. Petroleum 4:25-42. https://doi.org/10. 1016/j.petlm.2017.10.004

44. Wood DA (2019) Transparent open-box learning network provides auditable predictions for coal gross calorific value. Model Earth Syst Environ 5:395-419. https://doi.org/10.1007/s40808-0180543-9

45. Atkeson CG, Moore AW, Schaal S (1997) Locally weighted learning. Artif Intell Rev 11(1-5):11-73

46. Birattari M, Bontempi G, Bersini H (1999) Lazy learning meets the recursive least squares algorithm. Advances in neural information processing systems 11, pages 375-381. MIT Press, Cambridge, MA

47. Cover TM, Hart PE (1967) Nearest neighbor pattern classification. IEEE Trans Inf Theory 13(1):21-27

48. Fix E, Hodges Jr JL (1951) Discriminatory analysis, nonparametric discrimination: consistency properties. Technical report, USAF School of Aviation Medicine

49. Shakhnarovich G, Darrell T, Indyk P (2006) Nearest-neighbor methods in learning and vision: theory and practice (neural information processing), The MIT Press, ISBN:026219547X

50. Chen GH, Shah D (2018) Explaining the success of nearest neighbor methods in prediction. Found Trends Mach Learn 10(5-6):337588

51. Garcia S, Derrac J, Cano J, Herrera F (2012) Prototype selection for nearest neighbor classification: taxonomy and empirical study. IEEE Trans Pattern Anal Mach Intell 34(3):417-435

52. Gul A, Perperoglou A, Khan Z, Mahmoud O, Miftahuddin M, Adler W, Lausen B (2018) Ensemble of a subset of kNN classifiers. Adv Data Anal Classif 12(4):827-840. https://doi.org/10.1007/ s11634-015-0227-5
53. Samworth R (2012) Optimal weighted nearest neighbour classifiers. Ann Stat 40(5):2733-2763

54. Bontempi G, Birattari M, Bersini H (1999) Lazy learning for local modeling and control design. Int J Control 72(7/8):643-658

55. Lever J, Krywinski M, Altman N (2016) Model selection and overfitting. Nature methods 13: 703-704. Published online: https://doi.org/10.1038/nmeth.3968

56. Frontline Solvers (2019) Standard Excel Solver - Limitations of Nonlinear Optimization () https://www.solver.com/standard-excelsolver-limitations-nonlinear-optimization

57. Yang XS (2009) Firefly algorithms for multimodal optimization. Stochastic Algorithms: Foundations and Applications, SAGA, Lecture Notes in Computer Sciences 5792:169-178

58. Yang XS, He X (2013) Firefly algorithm: recent advances and applications. Int J Swarm Intell 1(1):36-50

59. Pal SK, Raj CS, Singh AP (2012) Comparative study of firefly algorithm and particle swarm optimization for Noisy non-linear optimization problems. I J Intell Syst Appl 10:50-57

60. Arora S, Singh S (2013) The firefly optimization algorithm: convergence analysis and parameter selection. Int J Comput Appl 69(3):48-52

61. Arora S, Singh S (2014) Performance research on firefly optimization algorithm with mutation. International conference on communication. Comput Syst: 168-172

62. Wood DA (2016a) Metaheuristic profiling to assess performance of hybrid evolutionary optimization algorithms applied to complex wellbore trajectories. J Nat Gas Sci Eng 33:751-768. https://doi. org/10.1016/j.jngse.2016.05.041

63. Wood DA (2016b) Evolutionary memetic algorithms supported by metaheuristic profiling effectively applied to the optimization of discrete routing problems, journal of natural gas science and engineering 35:997-1014. https://doi.org/10.1016/j.jngse.jngse.2016. 09.031

Publisher's Note Springer Nature remains neutral with regard to jurisdictional claims in published maps and institutional affiliations. 